Running title: Electron Cryo-Tomography of Axoneme Doublet Microtubule

\title{
Electron Cryo-Tomography Structure of Axonemal Doublet Microtubule from Tetrahymena thermophila
}

Sam Li ${ }^{1 *}$, Jose-Jesus Fernandez ${ }^{2}$, Amy S. Fabritius ${ }^{3}$, David A. Agard ${ }^{1}$ and Mark Winey ${ }^{3 *}$

1. Department of Biochemistry and Biophysics, University of California, San Francisco, CA 94158, USA.

2. Nanomaterials and Nanotechnology Research Center (CINN-CSIC), Health Research Institute of Asturias (ISPA), 33011 Oviedo, Spain.

3. Department of Molecular and Cellular Biology, University of California Davis, Davis, CA 95616, USA.

*Correspondence:

Sam Li (samli@ucsf.edu)

Mark Winey (mwiney@ucdavis.edu)

Key words: doublet microtubule, axoneme, flagella, motile cilia, Tetrahymena thermophila, evolution, electron cryo-tomography. 


\section{Summary}

Doublet microtubules (DMT) provide a scaffold for axoneme assembly in motile cilia. Aside from $\alpha / \beta$ tubulins, the DMT comprises a large number of non-tubulin proteins in the luminal wall of DMT, collectively named the microtubule inner proteins (MIPs). We used electron cryo-tomography to study axoneme DMT isolated from Tetrahymena thermophila. We present the structures of DMT at nanometer and sub-nanometer resolution. The structures confirm that MIP Rib72A/B binds to the luminal wall of the DMT by multiple DM10 domains, likely by recognizing the acetylated K40 residue of $\alpha$ tubulin. We found Fap115, a MIP containing multiple EF-hand domains, located at the interface of four-tubulin dimers in the lumen of the A-tubule. It functions as a "molecular staple" stabilizing both lateral and longitudinal tubulin interfaces and playing a critical role in DMT stability. Defects caused by the depletion of Fap115 propagate along the axoneme due to extensive structural changes in the DMT at and beyond the Fap1 15 binding site. Finally, by comparing DMT structures from Tetrahymena and Chlamydomonas, we have identified a number of conserved MIPs as well as MIPs that are unique to each organism. This conservation and diversity of the DMT structures might be linked to their specific functions. Our work provides structural insights essential for understanding the roles of MIPs during motile cilium assembly and function, as well as their relationships to human ciliopathies. 


\section{Introduction:}

The motile cilium is an evolutionarily conserved cellular organelle that has a wide range of biological functions. In protozoa, such as ciliates and green algae, motile cilia, also known as flagella, are essential for the cell's locomotion and feeding (Bornens, 2018). In metazoa, motile cilia have multiple functions. For example, in mammals, they are concentrated in the airway for mucus clearance, and in the ventricular tube of the brain for cerebrospinal fluid flow. In addition, motile cilia are found in the oviduct of female reproduction organs, in the male sperm and at the node of the gastrulation site during embryo development. Defective motile cilia cause a spectrum of human diseases, such as primary cilia dyskinesia and situs inversus (Reiter and Leroux, 2017).

In the core of a motile cilium, two singlet microtubules, known as the central pair $(\mathrm{CP})$, together with nine doublet microtubules (DMTs) that are radially surrounding the CP, establish a 9+2 configuration, a hallmark of the motile cilium. These microtubule-based structures provide a scaffold for assembly of hundreds of other ciliary components, such as the outer/inner dynein arms (ODA, IDA), the dynein regulatory complex (DRC), and the radial spokes (RS), all of which are essential for driving and regulating cilia beating. Fueled by ATP, the DMT-associated dynein generates power strokes that effectively slide one DMT relative to its adjacent DMTs. The continuous and asynchronous alternation of power stroke and sliding of DMTs on the opposite sides of the axoneme translates into a local bending of the cilium. This bending propagates along the cilium length manifested as various waveforms during ciliary beating (Lin and Nicastro, 2018; Mitchison and Mitchison, 2010). 
In addition to having an essential scaffold role during cilia beating, the DMT also serves as a bi-directional track for the intraflagellar transport (IFT) important for cilia assembly and maintenance (Rosenbaum and Witman, 2002). Kinesin motors drive anterograde transport moving on the B-tubule towards the plus end, while the cytosolic dyneins move in the opposite direction on the A-tubule for retrograde transport (Stepanek and Pigino, 2016).

The DMT comprises a full 13-protofilament (pf) A-tubule and an incomplete 10-pf Btubule that shares a wall, known as the partition, with the A-tubule. Besides $\alpha / \beta$ tubulins, it has a large number of proteins decorating the luminal wall of the A- and B-tubule, known as the microtubule inner proteins (MIPs) (Nicastro et al., 2011, 2006; Sui and Downing, 2006). Recent structure studies, in particular by cryoEM single particle analysis (SPA) and tomography (cryoET), have revealed the molecular architecture of the DMT in great details (Ichikawa et al., 2019, 2017; Khalifa et al., 2020; Ma et al., 2019; Song et al., 2020). These results showed that the MIPs nonuniformly decorate the luminal wall of the DMT. They form a tightly knitted, highly intricate meshwork bolstering the MT lattice. The structures highlighted the complexity of motile cilia as functional machinery and have substantially advanced our understanding of the composition of the axoneme in the context of its 3D structure. Meanwhile, these findings have raised many questions. For example, it is unclear precisely how the MIPs contribute to the function of motile cilia. Also, mechanistic detail on how these large numbers of MIPs are coordinated and their inter-dependence during the DMT assembly process is largely 
unknown. In addition, the structural conservation and diversity of the DMT in other

organisms is unclear. Here, we address many of these questions by reporting the cryoET results on the axonemal DMT isolated from Tetrahymena thermophila. In particular, we focused our analysis on the MIPs in the luminal wall of A- and B-tubules. 


\section{Results}

\section{Overall Structure of the DMT from Tetrahymena thermophila}

Previous studies show the MIPs decorate the Tetrahymena DMT wall asymmetrically, manifesting a range of longitudinal periodicities, from $8 \mathrm{~nm}$ up to $48 \mathrm{~nm}$ (Ichikawa et al., 2019; Song et al., 2020). In order to survey the entire structure at the highest possible resolution, we initially focused on the DMT with a $16 \mathrm{~nm}$ repeat. By aligning the A- and B-tubule separately, we obtained averaged maps of the DMT in an overall resolution of $10.2 \AA$ (Figure 1A, Figure S1, Table 1). In the average, in particular the MT backbones, the secondary structural elements can be resolved (Figure 1B). Meanwhile, many nontubulin proteins on the luminal wall of DMT can be discerned based on their overall shapes. These include Rib72 and Fap115 in the lumen of A-tubule, Fap20 and PACRG forming a filament at the inner junction, and Fap52, whose two WD40 domains each with characteristic seven $\beta$-propeller repeats can be resolved (Figure 1C). Meanwhile, a number of filamentous MIPs (fMIPs) associated at either inside or outside cleft between MT protofilaments can be resolved. Overall, the improved resolution facilitates identification of the MIPs in the DMT.

\section{Rib72A/B on the Luminal Wall of A-tubule}

The conserved Rib72 protein is an elongated multidomain MIP essential for flagella function and for recruiting other MIPs to the organelle (Fabritius et al., 2020; Ma et al., 2019; Stoddard et al., 2018). A human homolog of Rib72, EFHC1, is linked to juvenile myoclonic epilepsy (Gonsales et al., 2020; Suzuki et al., 2020, 2004). Based on the atomic model of Rib72 from Chlamydomonas, its N-terminus starts at pf A12 in the 
partition region. The molecule spans seven pfs in the luminal wall of the A-tubule. Its Cterminal domain (CTD) anchors at pf A05. While Chlamydomonas has a single copy of the RIB72 gene, Tetrahymena, like many other ciliates, possesses two paralogs, RIB72A and RIB72B, which share $41 \%$ identity. Both proteins have 3 tandem DM10 domains connected by flexible linkers. However, Rib72B lacks a C-terminal EF-hand motif. Previous studies showed that single or double knockouts of RIB72A and RIB72B in Tetrahymena caused extensive defects in the DMT and reduced cell motility (Stoddard et al., 2018). Here, we further investigated the binding of Rib72A/B to the MT wall and to other MIPs. By focusing subtomogram alignment on the pf A01 A06 region, the structure was improved slightly from 10.2 to $9.9 \AA$ (Figure 2 A, Figure S2, Table 1). In the average, Rib72A and B are distinguishable, alternating longitudinally every $8 \mathrm{~nm}$. In each Rib72A or B, three DM10 domains can be readily recognized binding to pfs A01, A03 and A05 respectively. We fit an atomic model of Rib72 from Chlamydomonas into the average map (Figure 2A). For Rib72B, the last 84 residues were removed, corresponding to the EF-hand motif. The models fit into the average map without any local modification, indicating that the topology of Rib72, including the DM10 domains and the length and curvature of corresponding linkers, are conserved between these two organisms (Figure 2A). This is also consistent with the previous observation that the sequences of each DM10 domain are more highly conserved between proteins from different species than they are within a particular molecule (King, 2006), suggesting each domain has unique and conserved functions. 
Like Chlamydomonas, each Rib72A and B uses three DM10 domains to anchor to the DMT wall. We built pseudo-atomic models for the corresponding pfs of the DMT. In the model, each DM10 domain binds at the longitudinal $\alpha / \beta$ tubulin interdimer interface. In the average, five out of six DM10 domains from Rib72A/B showed connecting density between the DM10 domain and the $\alpha$-tubulin (Figure 2B). Based on the models, these connections are between the last $\alpha$-helix in the DM10 domains and the H1-S2 loop in $\alpha$ tubulin, also known as the $\alpha \mathrm{K} 40$ loop. The loop is disordered in the undecorated MT (Eshun-Wilson et al., 2019; Howes et al., 2017; Zhang et al., 2015). Interestingly, the $\mathrm{K} 40$ residue is a potential site for post-translation modification by acetylation (Akella et al., 2010). Though at current resolution we could not resolve the lysine residue or the acetyl moiety in the structure, our observations corroborate the recent observations of the Rib72 in Chlamydomonas (Ma et al., 2019). The observed connecting densities between the DM10 domains and the $\alpha \mathrm{K} 40$-loops suggest that the Lysine (K40) residues in these $\alpha$ tubulins are acetylated. The Rib72A/B DM10 domains recognize and bind at the longitudinal interface of $\alpha / \beta$ tubulin at pfs A01, A03 and A05, and it is likely that one of the recognition features is the acetylated $\alpha \mathrm{K} 40$. Intriguingly, though the K40 acetylation is not essential for survival of Tetrahymena, depletion of K40 acetylation resulted in elevated MT dynamics (Akella et al., 2010; Gaertig et al., 1995). In addition to a direct mechanical role that the acetylated $\alpha$-tubulin might play by stabilizing the MT lattice during its bending (Eshun-Wilson et al., 2019; Xu et al., 2017), it is likely that the acetylK40 could also provide a binding site for recruiting MIPs, which will in turn further stabilize the DMT. 
In Chlamydomonas, the two longitudinally neighboring Rib72s are crosslinked at Rib72's N- and C-terminus by the MIPs Fap222 and Fap252 respectively (Ma et al., 2019). However, we could not find corresponding densities in the Tetrahymena structure.

Instead, we identified a previously unreported MIP at pf A04 A05 that is unique to Tetrahymena (Figure 2C, Video 1, 2). The MIP has an elongated bi-lobe shape, repeating every $16 \mathrm{~nm}$ in the longitudinal direction. It crosslinks the neighboring Rib72A and B in the luminal side of pf A05. At one end, a large globular domain contacts the EF-hand motif of Rib72A. A small domain directly binds to the DM10-3 domain of Rib72B. The two globular domains are connected by a funnel-shaped linker. Meanwhile, the MIP also makes multiple interactions with $\alpha / \beta$ tubulin dimer at pf A04 and with the linker region of Rib72A (Figure 2C). The estimated molecular weight of this MIP is $\sim 27 \mathrm{KDa}$.

\section{Binding of Fap115 on the Luminal Wall of A-tubule}

Recent studies showed that Fap115, a protein with multiple EF-hand motifs, was one of the MIPs missing in the RIB72 null mutations, suggesting the recruitment of Fap115 to the axoneme depends on Rib72 (Ma et al., 2019; Stoddard et al., 2018). Conversely, a Tetrahymena FAP115 knockout mutant retained Rib72A/B, suggesting Rib72's binding to the axoneme is independent of Fap115 (Fabritius et al., 2020). To further understand the molecular mechanism of Fap115 function, we used cryoET to study Fap115 in both wild-type and a FAP115 knockout mutant. In the wild-type, Fap115 binds to pf A02 A03, repeating every $8 \mathrm{~nm}$ in the longitudinal direction. Based on the models from Chlamydomonas, we built pseudo-atomic models of Fap115 and its neighboring Rib72A/B (Figure 3A). The model of Fap1 15 fits nicely into the map as shown by its 
secondary structure elements that could be clearly resolved. In our structure, Fap115 is shown as a bilobed structure with two domains, the N-terminal domain (NTD) and the Cterminal domain (CTD). They were connected by a flexible linker. Each domain has two EF-hands. The linker has an extended loop reaching out in the DMT plus-end direction making potential contact with the DM10-1 domain of Rib72 (Figure 3A). The NTD of Fap115 was anchored at the longitudinal interface of $\alpha / \beta$ tubulin heterodimer on pf A02 (the N-site, the non-exchangeable GTP site), potentially contacting both tubulins. The Fap115 CTD is particularly interesting. It is located at the junction of four-tubulin dimers interface, potentially interacting with all four tubulins (Figure 3B). It fits into an ideal space sandwiched between two $\beta$-tubulins from pf A02 and A03 (Figure 3C, Video 3). Specifically, the two H1-S2 loops from these $\beta$-tubulins resemble two jaws of a vernier caliper that contact the Fap115 CTD. Meanwhile, the S9-S10 loop from the A03 $\beta$ tubulin also interacts with the CTD. In addition to the two $\beta$-tubulins, the Fap115 CTD also makes several interactions with two $\alpha$-tubulins from pf A02 and A03. Notably, a loop of the EF-hand (EF-loop) extends towards the tubulin lateral interface, making potential contacts with the H1-S2/H2-S3 loops from A02 $\alpha$-tubulin and the M-loop from A03 $\alpha$-tubulin (Figure3D, Video 4). Due to the limited resolution in our structure, we could not directly observe side-chain interactions, therefore, caution has to be taken when interpreting the model. Nevertheless, the observed binding of Fap115 CTD at the interface of four tubulin dimers is reminiscent of the binding of the Calponin-homology $(\mathrm{CH})$ domain of the MT plus-end tracking EB proteins or the DC domain of doublecortin, a MT-stabilizing protein expressed in developing neurons. All these MAP domains preferentially bind to 13-pf MT and make contact to four neighboring tubulins on the 
outer surface of MT (Fourniol et al., 2010; Maurer et al., 2012; Zhang et al., 2015).

Although the details of the interaction are completely different, our observation indicated that Fap1 15 utilized a similar strategy as EBs or doublecortin for binding and stabilizing both longitudinal and lateral interfaces of MT lattice, specifically at the pf A02 and A03 interfaces, to help strengthen the DMT.

\section{FAP115 Knockout Results in Axoneme Defects Beyond Its Binding Site}

To further understand the function of Fap1 15 in motile cilia beating, we studied axonemes isolated from a FAP115 knockout strain (FAP115KO). Since Fap115 exhibits $8 \mathrm{~nm}$ longitudinal periodicity in the wild-type, in order to improve the resolution in the average, we limited the mutant axoneme length to $18 \mathrm{~nm}$ containing two repeats. We further focused the alignment on the pf A01 A06 region where the Rib72A/B and the Fap115 bind in the wild-type. The average of the FAP115KO mutant reached $9.6 \AA$ in resolution (Figure 4A, Figure S4A, Table 1). Compared to the wild-type, the bi-lobed structure of Fap1 15 is absent in the mutant (Figure 4B, Figure S4B), confirming the depletion of Fap115 in the mutant (Fabritius et al., 2020). However, Rib72A/B remain in the FAP115KO mutant structure (Figure 4B). This is in contrast to the Rib72 null mutants where the Fap115 was missing (Ma et al., 2019; Stoddard et al., 2018), suggesting the recruitment of Rib72A/B to the axoneme is independent of Fap115. Besides the absence of Fap115, we did not observe other defects in the mutant structure. For example, despite the Fap115 crosslinking of pf A02 A03 in the wild-type, we did not detect a change of curvature between pf A02 and A03 in the mutant. It is possible that the curvature or other structural change is subtle in the FAP115KO mutant and our current 
resolution is not high enough to detect the change. Alternatively, the DMT lattice is a robust assembly, and other MIPs might have overlapping function with the Fap115 in maintaining the DMT structural integrity.

We speculated that limiting our DMT segment length to $18 \mathrm{~nm}$ may have resulted in MIPs with longer periodicity, such as $48 \mathrm{~nm}$, being averaged out. To overcome this, we extended the segment length to $53 \mathrm{~nm}$ in subtomogram averaging, for both the wild-type and the FAP115KO (Figure S4G, H, Table 1). We further classified the averages, focusing on the partition region spanning pf A11 A13. For the FAP115KO mutant, we obtained two distinct classes from 9502 subtomograms, representing 60\% (5658 subtomograms) and 40\% (3844 subtomograms) of the population respectively (Figure 4C). Surprisingly, although the classification was restrained to the partition region, careful examination of these two classes revealed substantial structural differences extending beyond the partition region. First, there is a substantial loss of MIPs in the partition region in the $40 \%$ class (Figure 4D). Most notable is the loss of Rib43a-S/L, two fMIPs running longitudinally along the inner clefts between pf A11 A12 and A12 A13 respectively in the wild-type (Ichikawa et al., 2019). In addition, a number of MIPs in the partition, presumably associated with Rib43a-S/L, were also missing in this $40 \%$ class. Another structural difference is in the pf A04 A05 region. The 40\% class has substantial reduction of the aforementioned MIP density that longitudinally crosslinks Rib72A/B in the wild-type (Figures 4E, 2C). In contrast, the Rib72A/B remained present in both classes. Finally, a "tether density" at the inner junction is missing in the $40 \%$ class (Figure 4F). To rule out the possibility that these observed structural differences were 
caused by any artifact introduced during axoneme isolation, we examined the wild-type structure. The classification identified 772 (13\%) from 5855 subtomograms in the wildtype having defects in the partition region (Figure S4C, D). However, a close inspection on this minor class showed that other regions in DMT, including the pf A04 A05 and the inner junction region remain intact (Figures S4E, F). This is in contrast to the observation in the $40 \%$ class from the $F A P 115 \mathrm{KO}$ mutant where structural defects were identified in multiple locations. Taken together, this analysis demonstrated that the structural defects observed in the $40 \%$ of FAP $115 \mathrm{KO}$ mutant DMT were the consequence of the depletion of Fap115. It revealed that defects in the DMT due to the loss of Fap115 extended beyond its binding site at pf A02 A03.

The Rib43a-S/L are fMIPs in the partition region connected repeatedly in a head-to-tail fashion, forming two continuous filaments threading longitudinally throughout the axoneme. It is likely they provide a scaffold for other MIPs, binding and strengthening the local MT lattice. Since one of the major defects observed in the FAP115KO was the loss of Rib43a-S/L and other associated MIPs in the 40\% fraction of DMT, we asked if the defects would have any global impact on the axoneme structure. To address this question, we mapped the defective DMT identified by classification to the axoneme tomograms. The mapping of the two classes shows the defects were clustered in the mutant axoneme. Many formed long continuous stretches of defective DMT (Figure 4G). Given that many MIPs, in particular the fMIPs, such as Rib43a-S/L, form interwoven meshwork in the lumen of DMT, this is likely the result of a "domino effect" where a local structural defect affects its neighbor by propagating the abnormality along the DMT. 
In contrast to the mutant, the defective DMT detected in wild-type were dispersed and restricted to local sites in the axoneme as shown in the tomograms (Figure 4G). In summary, we revealed that depletion of Fap1 15 caused substantial defective DMT in the axoneme. The defect was not limited to the Fap115 binding site, instead, it spread to several regions in DMT. Furthermore, the defects were propagated along the DMT in axonemes of the FAP115KO mutant.

\section{Structural Comparison of MIPs in the DMT Between Tetrahymena and}

\section{Chlamydomonas}

The motile cilium is an evolutionarily conserved organelle (Mitchell, 2016; Satir et al., 2008). However, cilia from different organisms or species exhibit unique characteristics, ranging from differences in length, waveform, beating frequency and amplitude, and in response to various external stimuli. These differences are likely underlain by their unique molecular composition and structure (Satir et al., 2008). Previous studies have revealed marked differences in the cilia structure among different model organisms (Carvalho-Santos et al., 2011; Khalifa et al., 2020; Lin et al., 2014; Nicastro et al., 2011; Pigino et al., 2012). However, many comparisons were descriptive and were limited by the resolution of the structures. Recently, the structure of DMT in Chlamydomonas has been solved at near atomic resolution (Ma et al., 2019). In the light of these revelations, we sought to compare our DMT structure from Tetrahymena to the structure in Chlamydomonas, focusing on the MIPs. The comparison was not meant to be exhaustive, but by taking a heuristic approach we intend to learn the structure conservation and diversity of the DMT between these two model organisms that will infer their functions . 
Since the binding of MIPs to the DMT is not uniform, besides the aforementioned structural difference in the pf A01 A06 region, we chose to compare three additional regions clustered with MIPs. For each region, we took a set of MIP models from Chlamydomonas, used their relative distance and orientation as constraints and fit the models into our $48 \mathrm{~nm}$ DMT structure from the wild-type.

First, we compared the A-tubule seam region spanning pf A06 A10. This region is populated by MIPs with 48-nm periodicity. We found two fMIPs in our map, corresponding to Fap53 and Fap127 from Chlamydomonas (Figure 5A). The models fit into our map very well, including the local kinks and bulges of the filaments. In particular, both Fap53 and Fap127 turned 90 degrees at the expected positions, transitioning from longitudinal to transverse direction across the seam towards the outer junction, indicating their high degree of structural conservation. In addition, we also found two copies of Fap67, a nucleoside diphosphate kinase. The shapes and locations are consistent with the structure in Chlamydomonas. Lastly, we found a likely density for Fap161, a MIP binding at the A-tubule seam. However, its EF-hand motif had to be adjusted to better fit into our map (Figure 5A). Surprisingly, there were at least six globular densities in our map that we could not find their corresponding models in Chlamydomonas DMT. Five were in the seam region, including two globular densities previously assigned as MIP2b c (Song et al., 2020). Another density laterally extended from pf A05 to A07 (Figure 5A). Conversely, we could not fit eight MIP models from Chlamydomonas into our map. These MIPs are: Fap182, Fap129, Fap85, Fap21, Fap68, Fap143, Fap95, 
Fap141 (Figure S5A) . Likely, this is in part due to the limited resolution of our map and the unstructured nature of the extended MIPs, such as Fap129 and Fap143.

Next, we examined the pf A10 A01 region, including the partition wall shared by the Aand B-tubule. Recent single particle cryoEM studies have identified two filamentous structures in the partition, running longitudinally on the cleft between pf A11/A12 and A12/A13 respectively (Ichikawa et al., 2019; Ma et al., 2019). Each filament is composed of multiple copies of Rib43a connected in a head-to-tail fashion. In Chlamydomonas, the filaments in both positions were composed of Rib43a. However, Tetrahymena has two isoforms, RIB43a-S and RIB43a-L. While the filament at A12/A13 was composed solely of Rib43a-S, the filament at A111/A12 was composed of alternation of Rib43a-S and Rib43a-L (Ichikawa et al., 2019). We confirmed this by fitting both structures in our map (Figure 5B). Besides Rib43a-S/L, the two organisms displayed marked differences in this region. We could not find models for six globular densities that were associated with Rib43a-L/Rib43a-S filament (Figure 5B). Five of them have been assigned previously as MIP4a e (Song et al., 2020). Meanwhile, five MIPs from Chlamydomonas, Fap166, Fap273, Fap363, Rib21, Rib30, could not find corresponding density in our structure (Figure S5B). Given the difference in Rib43a between these two organisms and that many MIPs were directly associated with the Rib43a, the observed structural differences are not completely surprising.

Lastly, we examined the inner junction region. Previous studies showed structural differences between Tetrahymena and Chlamydomonas in this region (Khalifa et al., 
2020; Ma et al., 2019). Our modeling study confirmed this. We identified Fap52 and Fap106 in our map (Figure 5C). However, our structure does not show densities corresponding to Fap126 and Fap276. Conversely, we found a second "tether density" linking Fap52 to pfA13 and a 16-nm periodic structure on the ridge of pf A12 facing the lumen of B-tubule (Figure 5C). Both are unique to Tetrahymena. Previously the MIP on pf A12 was assigned as MIP5 (Song et al., 2020).

Surprisingly, we uncovered a MIP at the Tetrahymena inner junction that was not found in Chlamydomonas. Previously, this MIP was indicated as a minor density (Ichikawa et al., 2017; Song et al., 2020). However, by multiple iterations of classification and alignment, we show this MIP is in fact a large multi-domain protein or complex, with an estimated molecular weight of $\sim 130 \mathrm{KDa}$. It has an overall X shape with 48-nm longitudinal periodicity. It spans multiple pfs across the inner junction, from B07 to A13. Longitudinally, it is $\sim 16 \mathrm{~nm}$ wide (Figure 6A, Video 5, Figure S6). To further characterize the interaction of this MIP with other components in the inner junction, we fit models of Fap52, Fap45 and Fap210, into our density map (Figure 6B, Video 6). This MIP contacts the models at multiple points. Specifically, it binds to the Fap210 at pf B06/B07, it also binds to two copies of Fap45 on the clefts between pf B07/B08 and B08/B09 (Figure 6B, Video 6). In addition, the MIP interacts with Fap106 and pf A13. Interestingly, the MIP contacts Fap52 via Fap45. The N-terminal helix of Fap45 threads through a narrow gap between this MIP and the Fap52, making contacts with both. This Fap45 has a kink at this point; it continues and makes a $\sim 90$-degree bend into the transverse direction where it interacts with a second molecule of Fap45. When the helix 
of Fap45 in the transverse direction reaches the inner cleft of pf B07/B08, it takes a second 90-degree sharp bend, switching back to the longitudinal direction towards the plus end. At this point, it contacts the C-terminal end of a third Fap45 molecule (Figure 6B), forming a longitudinal head-to-tail connection, a common theme shared by many fMIPs in the axoneme (Ichikawa et al., 2019; Ma et al., 2019). To find out if this MIP had any preferential binding to any particular DMT, we mapped the structure on the axonemes. We could not detect any radial asymmetry or any DMT preference, instead, this MIP was found in all 9 DMTs in the axoneme. Taken together, we have identified a large MIP complex at the inner junction that is unique to the Tetrahymena DMT. It makes extensive interactions with multiple MIPs and the luminal wall of DMT. In the future, it will be interesting to identify the molecular nature of the MIP and to understand its function. 


\section{Discussion}

\section{Fap115 Functions as a "Molecular Staple" Essential for Structural Integrity of the DMT}

The EF-hand protein Fap1 15 was identified as one of the MIPs lost in the Rib72A/B knockout mutants (Fabritius et al., 2020; Ma et al., 2019; Stoddard et al., 2018). In this study, we provide a detailed description of Fap115 binding to the DMT: The protein bridges pf A02 and A03 on the luminal wall of the A-tubule, making multiple interactions with the adjacent proteins; its NTD interacts with $\alpha / \beta$ tubulin in pf A02. The CTD anchors at the corner of four-tubulin dimers, stabilizing both lateral and longitudinal MT interfaces at pf A02 and A03. In addition, Fap115 interacts with either Rib72A or Rib72B via an extended loop. We further analyzed the axonemes isolated from a FAP115KO mutant. Strikingly, compared to the wild-type, $40 \%$ of DMT showed structural defects beyond the Fap115 binding site. The defects were identified in the pf A04 A05 region, the partition region and the inner junction region. Moreover, these defects propagated forming long stretches of defective DMT in the axoneme, and likely causing the motility defect observed in the mutant (Fabritius et al., 2020). Interestingly, despite extensive interactions between Fap115 and the luminal wall of DMT, Fap115 was absent in the Rib72 null mutations (Ma et al., 2019; Stoddard et al., 2018), suggesting $\mathrm{Rib} 72 \mathrm{~A} / \mathrm{B}$ are essential for recruitment of Fap115 to the axoneme and their binding might take place prior to Fap115 assembly on the axoneme. Conversely, we did not observe any significant reduction of $\mathrm{Rib} 72 \mathrm{~A} / \mathrm{B}$ in the $F A P 115 \mathrm{KO}$ mutant. Taken together, these findings, consistent with previous studies on the other part of axoneme, suggests that the 
axoneme assembly is a sequential and tightly controlled process (Diener et al., 2011; Qin et al., 2004).

Perhaps one of the most striking and interesting findings in the mutant is the extensive defects observed beyond the Fap115 binding site in the DMT and the propagation of the defects along the axoneme. This is likely a secondary effect caused by the Fap115 depletion. A similar effect has been observed previously with depletion of other MIPs. For example, in Chlamydomonas it was found that loss of inner junction components PACRG and Fap20 caused reduction of the beak and the IDA structure that were distant from the inner junction (Dymek et al., 2019; Yanagisawa et al., 2014). Currently, we lack knowledge on the timing of when these defects occurred in the axoneme. It could take place during cilia assembly in the FAP115KO mutant where the absence of Fap115 might affect recruitment of other MIPs. More likely the defects were introduced during cilia beating when the axoneme experienced substantial mechanical stress. Since the MIPs bind to DMT nonuniformly and the axoneme DMT are in a radially asymmetric shape, the curvatures between laterally associated pfs vary and might be finely tuned by the associated MIPs (Ichikawa et al., 2019, 2017) . Notably, besides the seam region, the pf A02/A03, where Fap115 binds, has the highest local inter-protofilament curvature in the DMT, a specific geometry that fits Fap115 well. During cilia beating, a disruption of the local curvature or geometry due to the depletion of MIP(s), such as Fap115, could have a profound and long-range effect on the DMT structure. This is consistent with previous in vitro experiments on MT, where local defects can have a long-range effect on distant sites (Rai et al., 2021; Schaedel et al., 2015). 
Microtubules and axonemes are intrinsically stiff polymers (Sale and Satir, 1976;

Schaedel et al., 2015). However, local bending and flexibility are necessary for generating waveform. Therefore, there exists a fine balance between the microtubule's intrinsic stiffness and the plasticity necessary for local bending and sliding during cilia beating. It is likely that one of the roles for many MIPs, such as Fap115, is to fine-tune the physical properties of MT, and to insulate any defect introduced under mechanical stress, preventing the defects from propagating. Overall, we propose that Fap115 functions as a "molecular staple" strengthening the local DMT lattice by bolstering interand intra-protofilament interfaces, making DMT more resilient to defects introduced during cilia beating.

\section{Structural Conservation and Diversity of MIPs in Two Model Organisms}

Tetrahymena and Chlamydomonas represent two phylogenetically distant groups: ciliates and green algae, which were estimated to have diverged more than a billion years ago (Kumar et al., 2017).

Remarkably, the axoneme of the motile cilium/flagellum is one of the most conserved structures during the evolution of the eukaryotic branches of the phylogenetic tree of life (Bornens, 2018; Carvalho-Santos et al., 2011; Cavalier-Smith, 2002; Mitchell, 2016; Satir et al., 2008; Satir and Christensen, 2007). In addition to comparative genomic and proteomic analyses in different organisms (Avidor-Reiss et al., 2004; Fabritius et al., 2020; Li et al., 2004; Ostrowski et al., 2002; Pazour et al., 2005; Smith et al., 2005), 
structural comparisons of cilia among different organisms have been carried out previously, showing both evolutionary conservation and diversity of this organelle (Carbajal-González et al., 2013; Khalifa et al., 2020; Lin et al., 2014; Nicastro et al., 2011; Pigino et al., 2012). However, most studies were limited to morphological descriptions, partly due to the limited resolution in the structures. Here, in the light of the improved resolution in the axoneme structures, we take an initial step to systematically examine the presence of MIPs in axonemes from Chlamydomonas and Tetrahymena. Our goal is to reveal structural conservation and diversity of the MIPs and to have a deeper understanding of their functions.

The results are summarized in Table 2. In the study, we found 19 MIPs that were shared by both organisms. Interestingly, among these 19 MIPs, except Fap112, Fap115 and DC3, 16 have found their human orthologue proteins, 13 are ciliopathic in humans or cause phenotypic defects in other metazoan organisms. This indicates that these MIPs are evolutionarily conserved and have critical functions in motile cilia in many organisms or species. For example, Fap53, Fap127, and Fap161 are near the seam of the A-tubule (pf A09 A10), which might provide critical structural roles. PACRG, Fap20, Fap52, and Fap106 are components of the inner junction and essential for its structural stability (Dymek et al., 2019; Khalifa et al., 2020; Ma et al., 2019; Owa et al., 2019; Yanagisawa et al., 2014). Fap67, a nucleoside diphosphate kinase that binds to the DMT via its DM10 domain, has been postulated to have a role in maintaining the local GTP concentration that is essential for axonemal homeostasis (Ma et al., 2019). Rib72A/B and Rib43a-S/L, which span several protofilaments laterally or form head-to-tail longitudinal filaments, 
might have critical roles in structural stability of the DMT as well as providing binding scaffolds for other MIPs (Norrander et al., 2000; Stoddard et al., 2018). CCDC39/Fap59 and CCDC40/Fap172 form a molecular ruler, essential for establishing and maintaining the 96-nm periodicity of the axoneme (Oda et al., 2014). Given the structural conservation of these MIPs that we observed and the phylogenic distance between Tetrahymena and Chlamydomonas, these are likely to be a set of ancestral MIPs that had been present in the last eukaryotic common ancestor (LECA) (Mitchell, 2016).

Meanwhile, we also found 19 MIPs or densities that are unique to either Tetrahymena or Chlamydomonas, showing marked differences in DMT structures between these two organisms. The morphological differences of axonemes among different evolutionary groups have been noticed previously. It has been implied that the structural differences might be linked to the variation of waveform (Kirima and Oiwa, 2017; Pigino et al., 2012). Interestingly, the flagella in Tetrahymena adapted an asymmetric planar whip-like waveform, while Chlamydomonas has both a planar whip-like waveform in forward locomotion and a sinusoidal-like symmetric waveform when swimming backwards. It will be intriguing to speculate if any subset of these MIPs might attribute to a particular type of waveform.

Taken together, by comparing the DMT structures from two model organisms, Tetrahymena and Chlamydomonas, we have identified a group of MIPs that are present in both structures as well as subsets that are unique to each organism, showing their structural conservation and diversity. In the future it will be interesting to expand this 
bioRxiv preprint doi: https://doi.org/10.1101/2021.07.13.452106; this version posted July 14, 2021. The copyright holder for this preprint (which was not certified by peer review) is the author/funder. All rights reserved. No reuse allowed without permission.

comparative structure study to other organisms and to gain insight into the function of this ancient and remarkable organelle in the course of evolution. 


\section{Acknowledgement}

We thank Zanlin Yu and David Bulkley (UCSF) for assistance on tomography data collection, Wynton HPC team at UCSF for supporting the computational infrastructure, Daniela Nicastro (UT, Southwestern) for kindly providing a Tetrahymena axoneme purification protocol, Tom Goddart (UCSF) for help with UCSF Chimera software. Fei Guo (UC, Davis) for help preparing cryo-grids. We are grateful to many of our colleagues for critical reading of the manuscript and for their encouragements. This work is supported in part by NIH grants R01GM127571 (M.W.), R35GM118099 (D.A.A.), and by the Spanish AEI/FEDER (SAF2017-84565-R) (J.-J.F.).

The EM structures have been deposited in the EMDB with the following accession numbers: EMD-24364, EMD-24366, EMD-24367, EMD-24368, EMD-24370, EMD24371, EMD-24372, EMD-24373, EMD-24374, EMD-24375, EMD-24376, EMD-24377, EMD-24379, EMD-24380, EMD-24381, EMD-24382.

\section{Declaration of Interests}

The authors declare no competing interests. 


\section{Figures and Figure Legends}

\section{Figure 1}

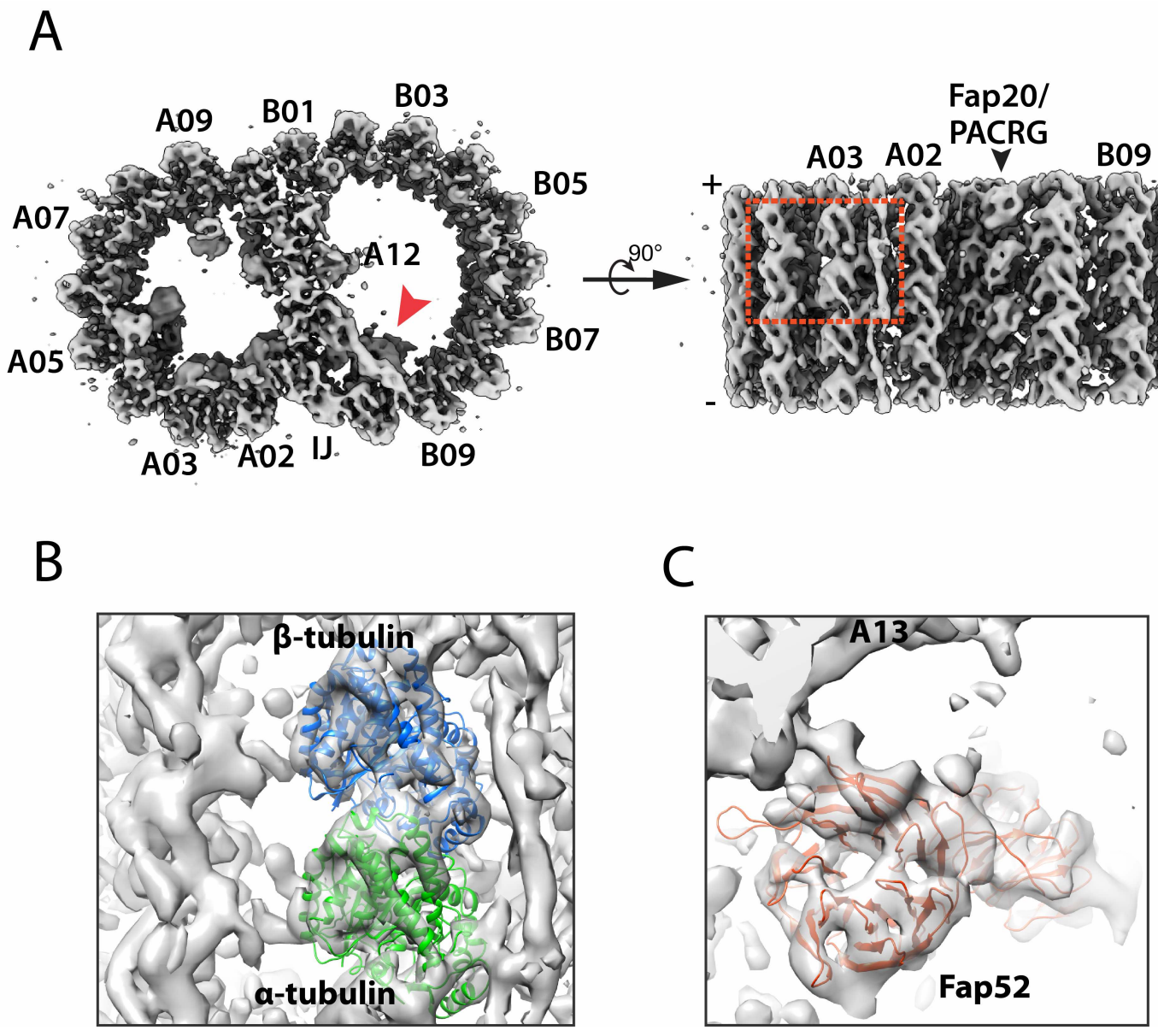

Figure 1. Structure of the 16-nm Repeat of the Doublet Microtubule from the Wildtype

A. The DMT structure is displayed as iso-surface representation in two orthogonal views.

Left, the DMT is viewed from the plus end. IJ: inner junction.

Right, the DMT is viewed from the lumen of axoneme. 
B. A magnified view of the boxed region in (A) shows that the secondary structure elements can be resolved in many parts of the map. Atomic models of $\alpha$ (green) and $\beta$ (light blue) tubulin are fit into the protofilament A03 in the map.

C. An atomic model of Fap52, one of the MIPs at the inner junction whose location in the DMT is indicated by a red arrowhead in (A), is fit into the average map. The blades of the $\beta$-propeller fold from the Fap52 WD40 domain are resolved in the map.

\section{Figure 2}

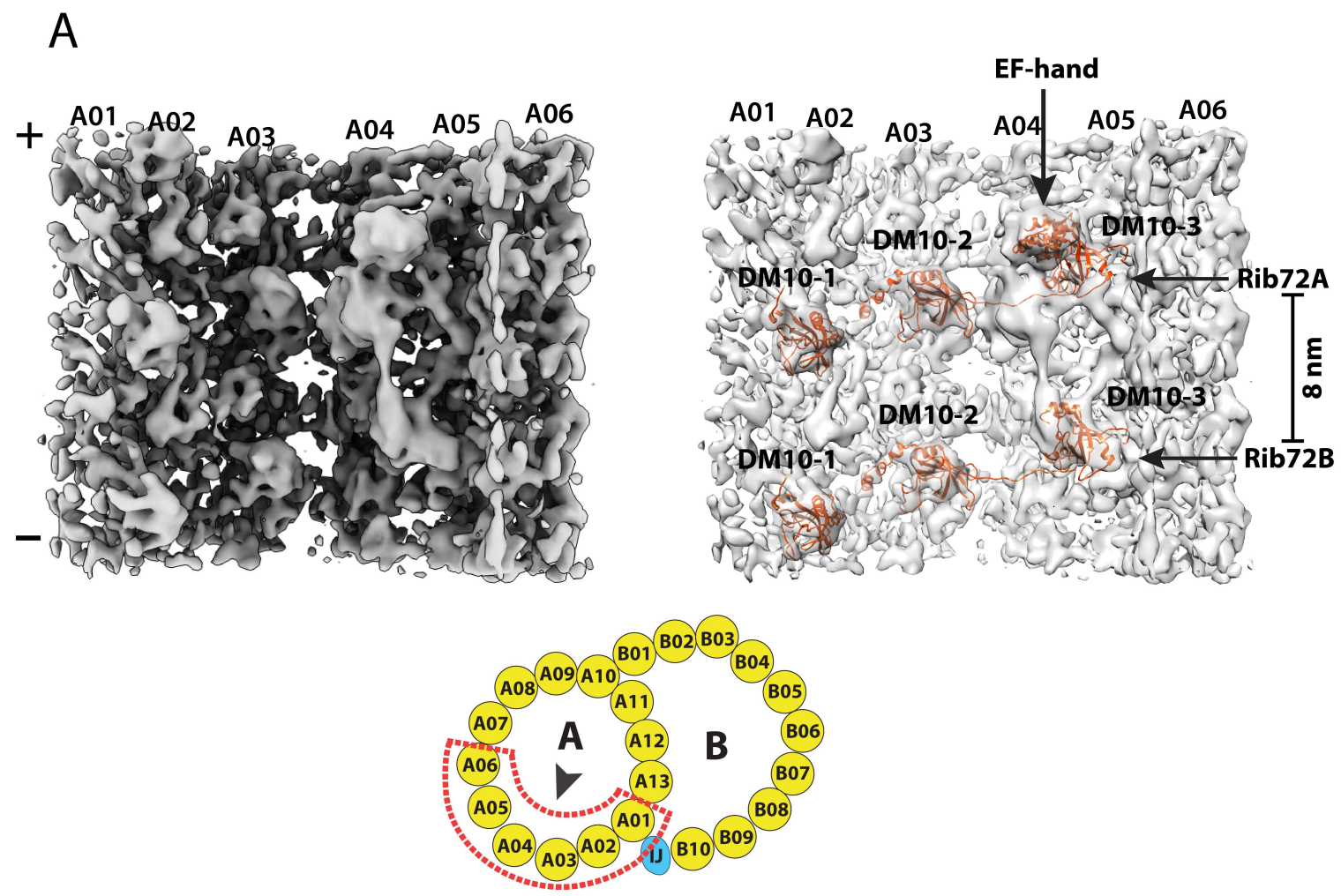



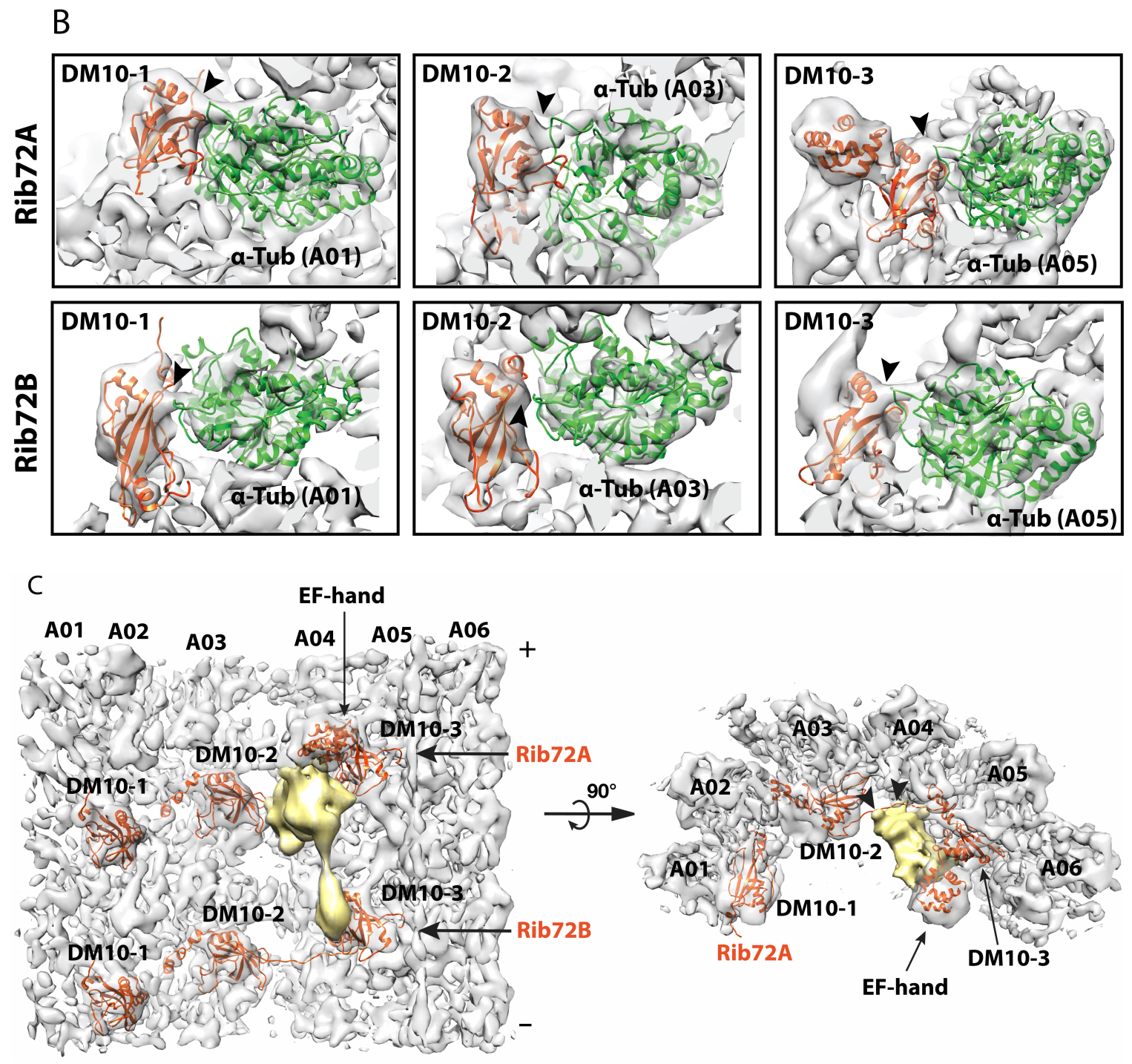

Figure 2. Structure of the 16-nm Repeat of the Doublet Microtubule Focusing on the

\section{Rib72A and Rib72B Binding Region}

A. A sideview of the density map focusing on the Rib72A/B binding sites. Left: the map is viewed from the luminal side of DMT as indicated by an arrowhead in the cartoon. Right: pseudo-atomic models of Rib72A and Rib72B, based on the structure from Chlamydomonas, are fit into the density map. The six DM10 domains and an EFhand motif in the Rbi72A C-terminus are indicated. In the cartoon. The red dashed 
lines outline the structure feature used for focused local refinement. This includes the protofilaments A01 A06 and their associated MIPs.

B. The DM10 domains bind to the K40 loops at the luminal side of $\alpha$-tubulin. The models for the DM10 domains ( in red) are fit into the density map. The $\alpha$-tubulins are in green. The black arrowheads indicate the connecting densities and the potential interactions between the DM10 domain and the K40 loop from $\alpha$-tubulin. The top row shows three DM10 domains from Rbi72A. The bottom row shows three DM10 domains from Rib72B.

C. An unidentified MIP (in gold) crosslinks the C-terminus of Rib72A and Rib72B. The averaged density map is shown in two orthogonal views. The black arrowheads indicate the binding sites of the unidentified MIP to the linker between DM10-2 and DM10-3 from Rib72A and to protofilament A04.

\section{Figure 3}
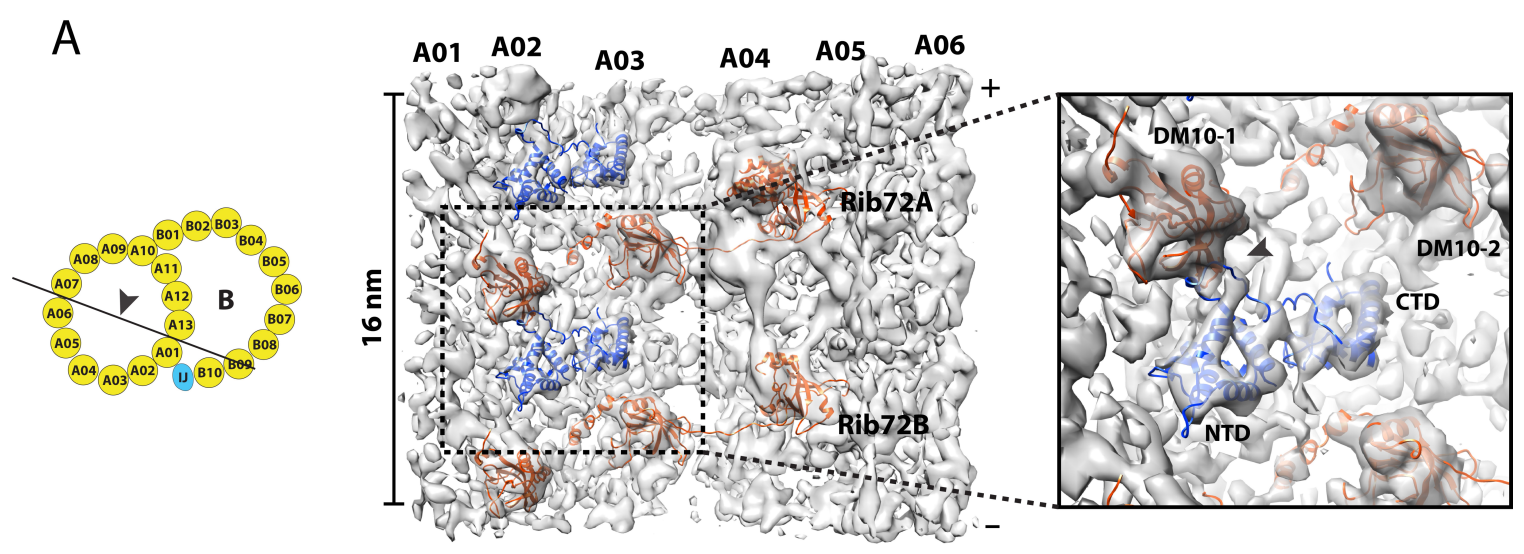

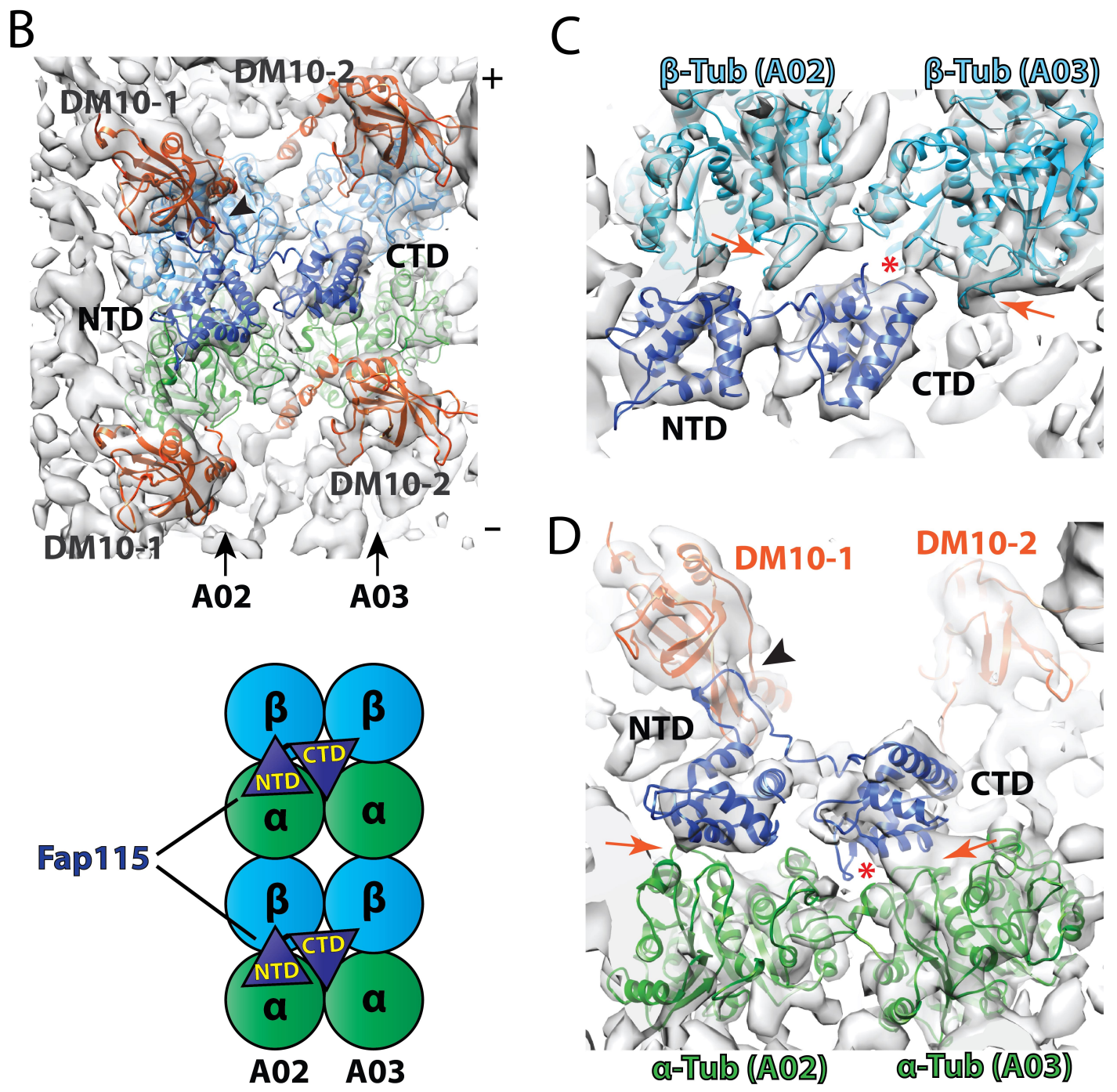

Figure 3. Binding of Fap115 to the Luminal Wall of Doublet Microtubule

A. Fap1 15 binds to protofilament A02 and A03 in the lumen of A-tubule. The atomic models for Fap115 (dark blue) and Rib72A/B (red) are fit into the density map. The viewing direction of the map is depicted in a cartoon on the left. The inset on the right shows a magnified view of Fap115. An arrowhead in the inset indicates the potential interaction between an extended loop from Fap115 and the first DM10 domain (DM10-1) from Rib72A that is anchored at protofilament A01. 
B. Binding of Fap115 to protofilament A02 and A03. The density map is shown in the same viewing direction as in (A). Two pairs of $\alpha / \beta$ tubulin dimers are fit into the density map, $\alpha$-tubulins are in green, $\beta$-tubulins are in light blue. The two EF-hand domains from Fap1 15, the N-terminal domain (NTD) and the C-terminal domain (CTD), are in dark blue. The cartoon illustrates the binding of Fap115 to two pairs of $\alpha / \beta$ tubulin dimers from pf A02/A03.

C. Potential interactions between Fap115 and two $\beta$-tubulins from pf A02/A03. The H1S2 loops from two $\beta$-tubulins are indicated by the red arrows. These two loops resemble two jaws of a vernier caliper where Fap115 CTD fits in and makes contacts. A red asterisk indicates potential interaction between Fap115 CTD and the S9-S10 loop from A03 $\beta$-tubulin, $\mathrm{C} \alpha$ - $\mathrm{C} \alpha$ distance $<7 \AA$.

D. Potential interactions between Fap115 and two $\alpha$-tubulins from pf A02/A03. The two red arrows indicate potential interaction interface between Fap115 NTD, CTD and two $\alpha$-tubulins, $\mathrm{C} \alpha$ - $\mathrm{C} \alpha$ distance $<7 \AA$. A red asterisk indicates an EF-hand loop from Fap1 15 that extends towards the lateral interface between two $\alpha$-tubulins, making potential interactions. 


\section{Figure 4}

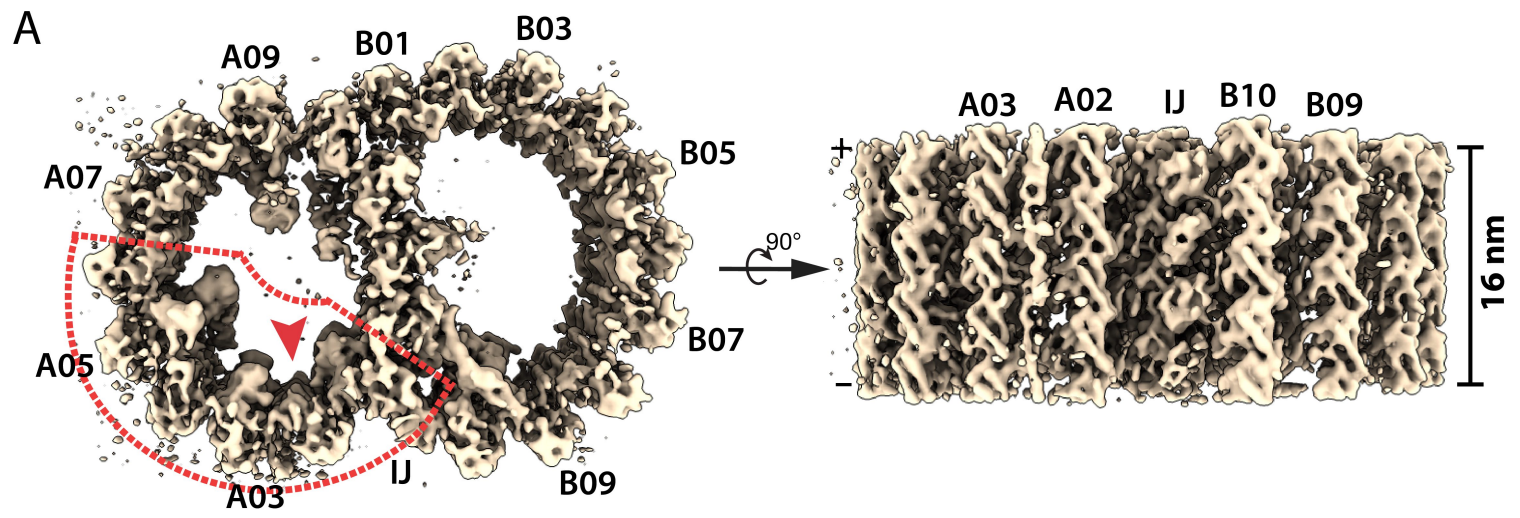

B

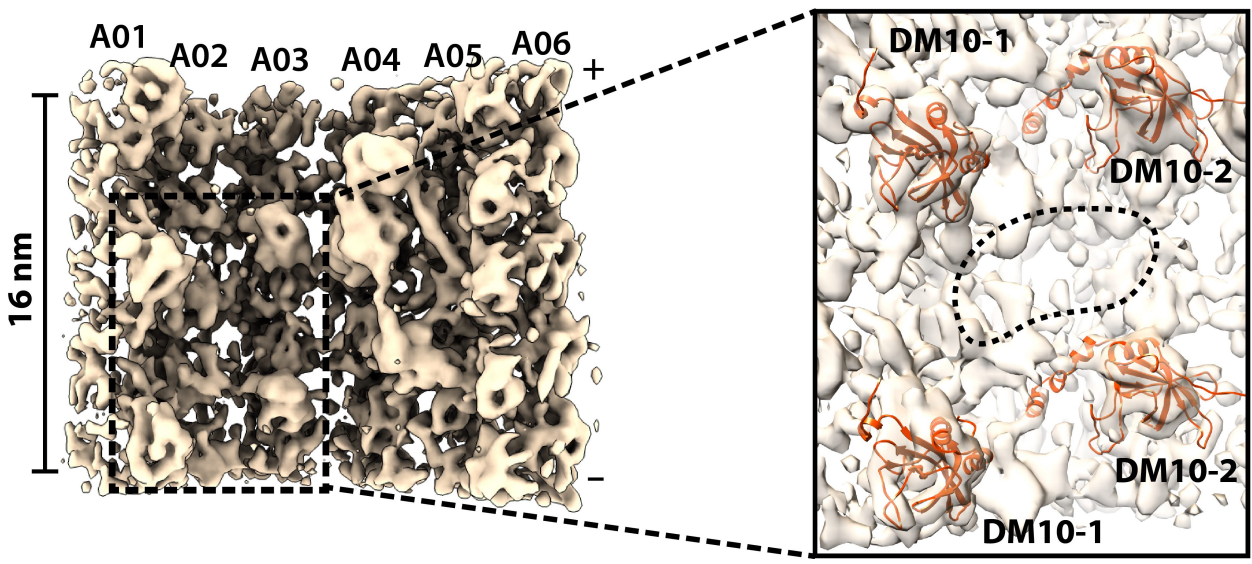

C
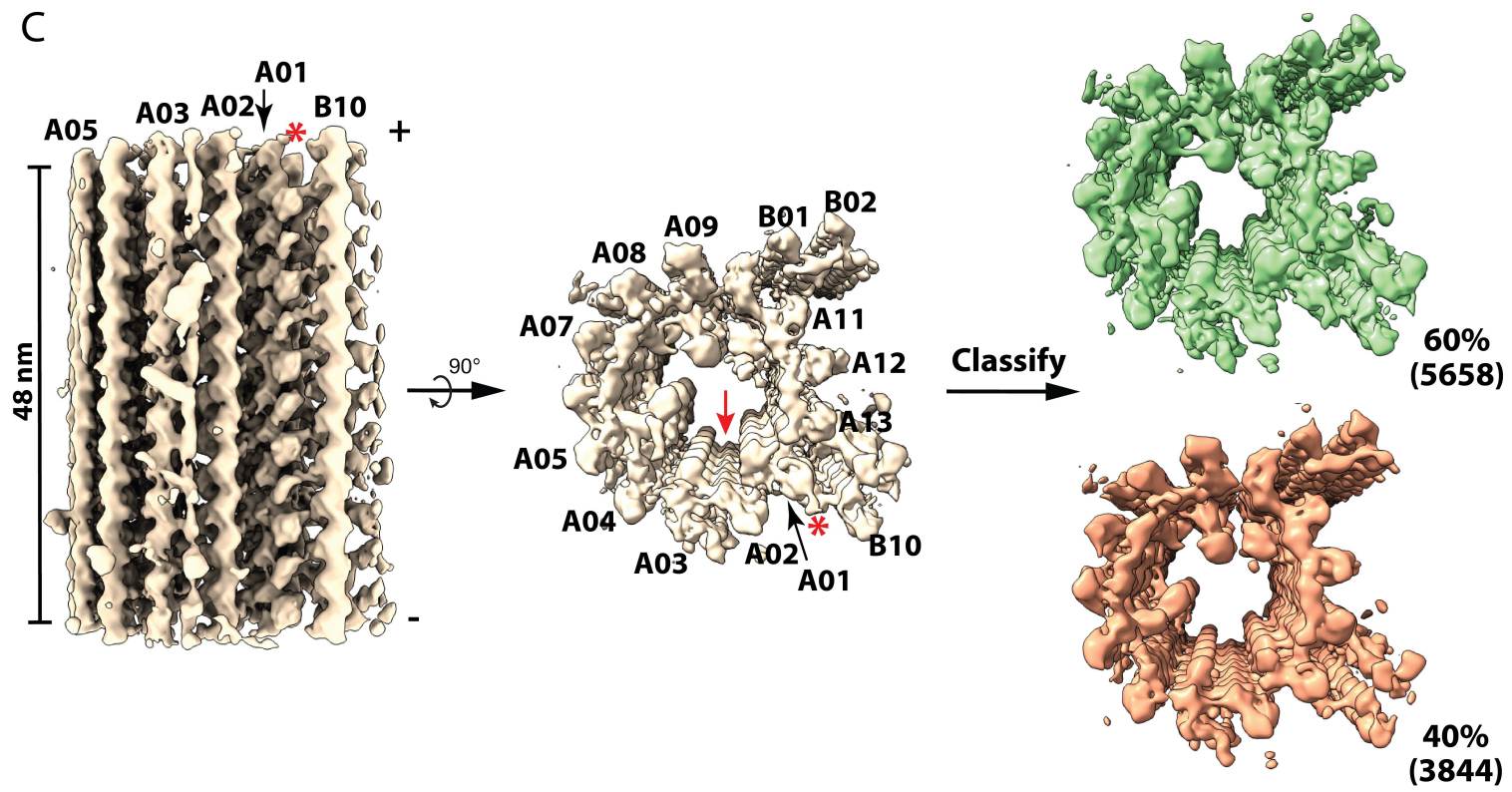

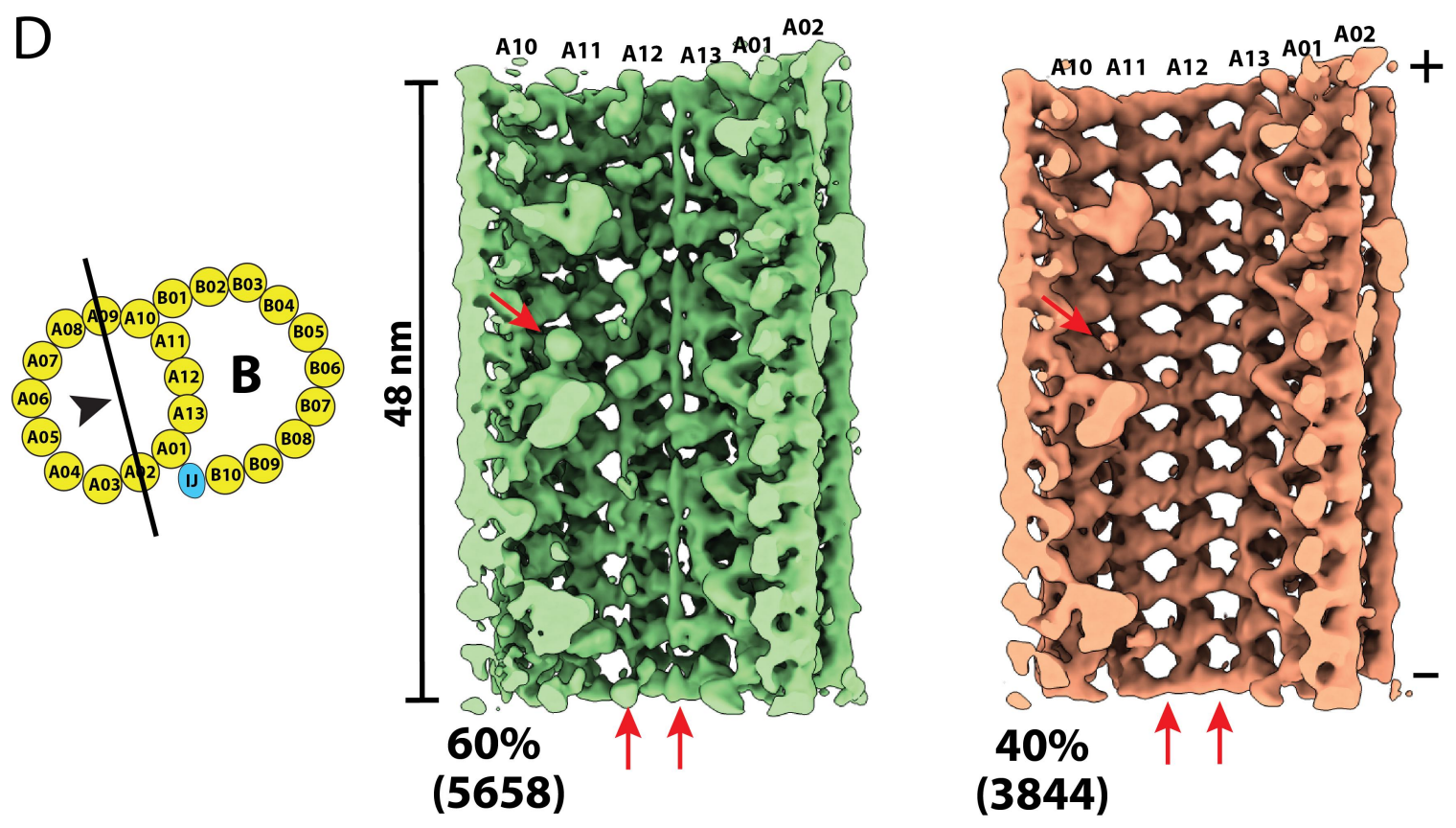

E
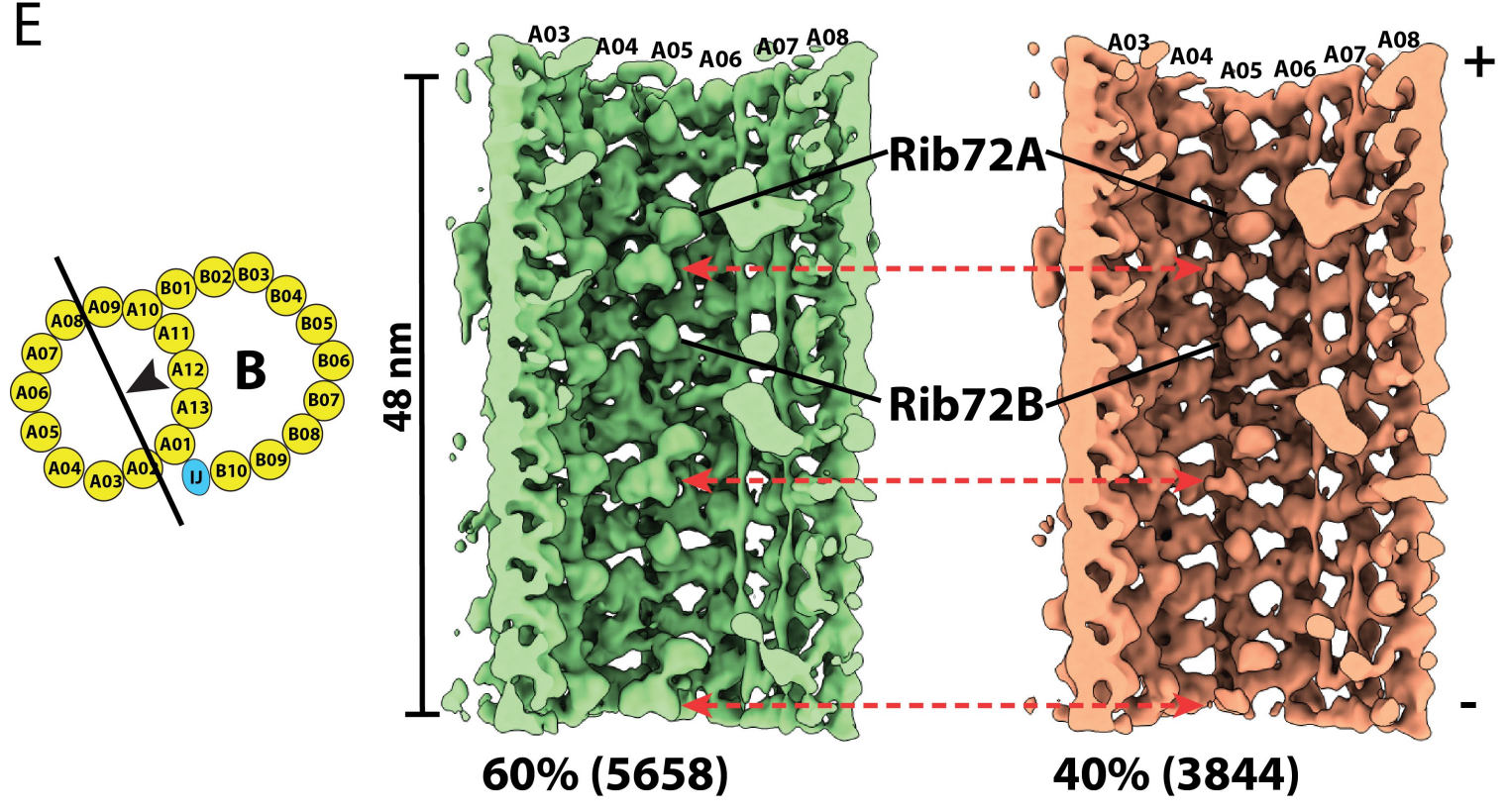

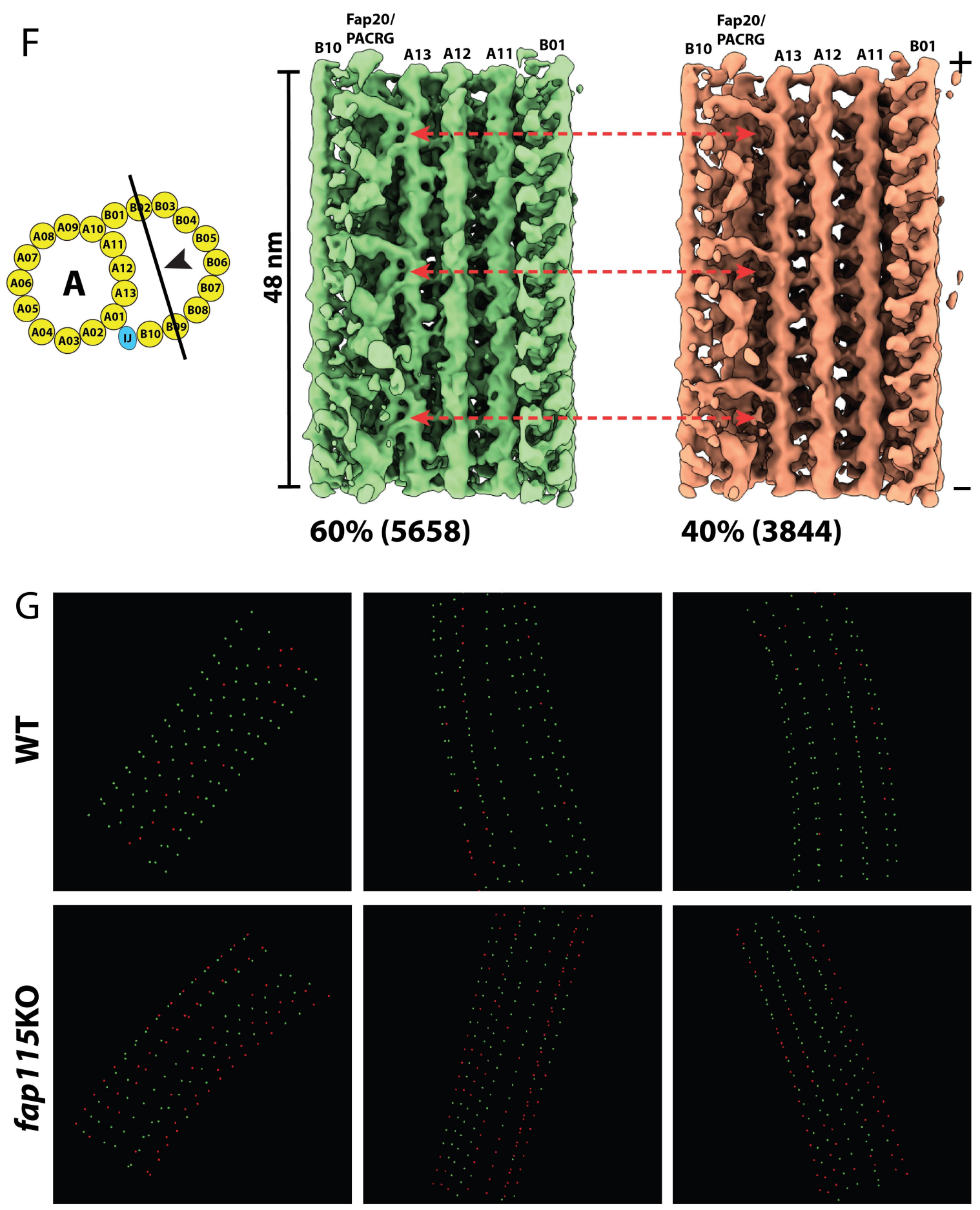

Figure 4. Structures of the Doublet Microtubule from FAP115 Knockout Mutant

A. Structure of the 16-nm repeat of the doublet microtubule from the FAP115KO mutant.

Two orthogonal views of the structure are shown. A red arrowhead indicates the location of Fap115 in the wild-type and the viewing direction in (B). The red dashed 
lines outline the region of A-tubule centered on Fap115 that was used in the focused refinement. The refinement result is shown in (B).

B. Structure of part of A-tubule from FAP115KO mutant viewed from the lumen. The inset shows a magnified view of the boxed area. The models for Rib72A/B (red) are fit into the density map. The DM10 domains are indicated. The dashed oval circle highlights the location of Fap115 in the wild-type but it is empty in the FAP115KO mutant structure.

C. Classification of the 48-nm Repeat of the A-tubule from the FAP115KO Mutant. The structure is shown in two orthogonal views. A red arrow indicates the expected location of Fap115 in the wild-type. A red asterisk indicates the inner junction protofilament composed of PACRG and Fap20. The classification focusing on the partition pf A11 A13 resulted in two classes as shown on the right - the $60 \%$ intact class in green and the $40 \%$ defective class in orange. The number of subtomograms in each class is indicated in parenthesis.

D, E, F Comparison of the two class-averages reveals major structure differences in three regions of the DMT, the partition region, the A04A05 region and the inner junction region. The viewing directions are depicted by the black arrowheads in the cartoons. The two class-averages are displayed side-by-side. The red arrows highlight the structure differences in each region.

G. Mapping the intact and the defective DMT structures in the axoneme. Three representative tomograms from the wild-type and the FAP115KO mutant are shown. Each green or red point represents a 48-nm segment of the DMT, green: intact 
structure. red: defective structure. The continuous dotted line represents the DMT.

Each dataset has 9 dotted lines representing 9 DMTs in the axoneme.

\section{Figure 5}
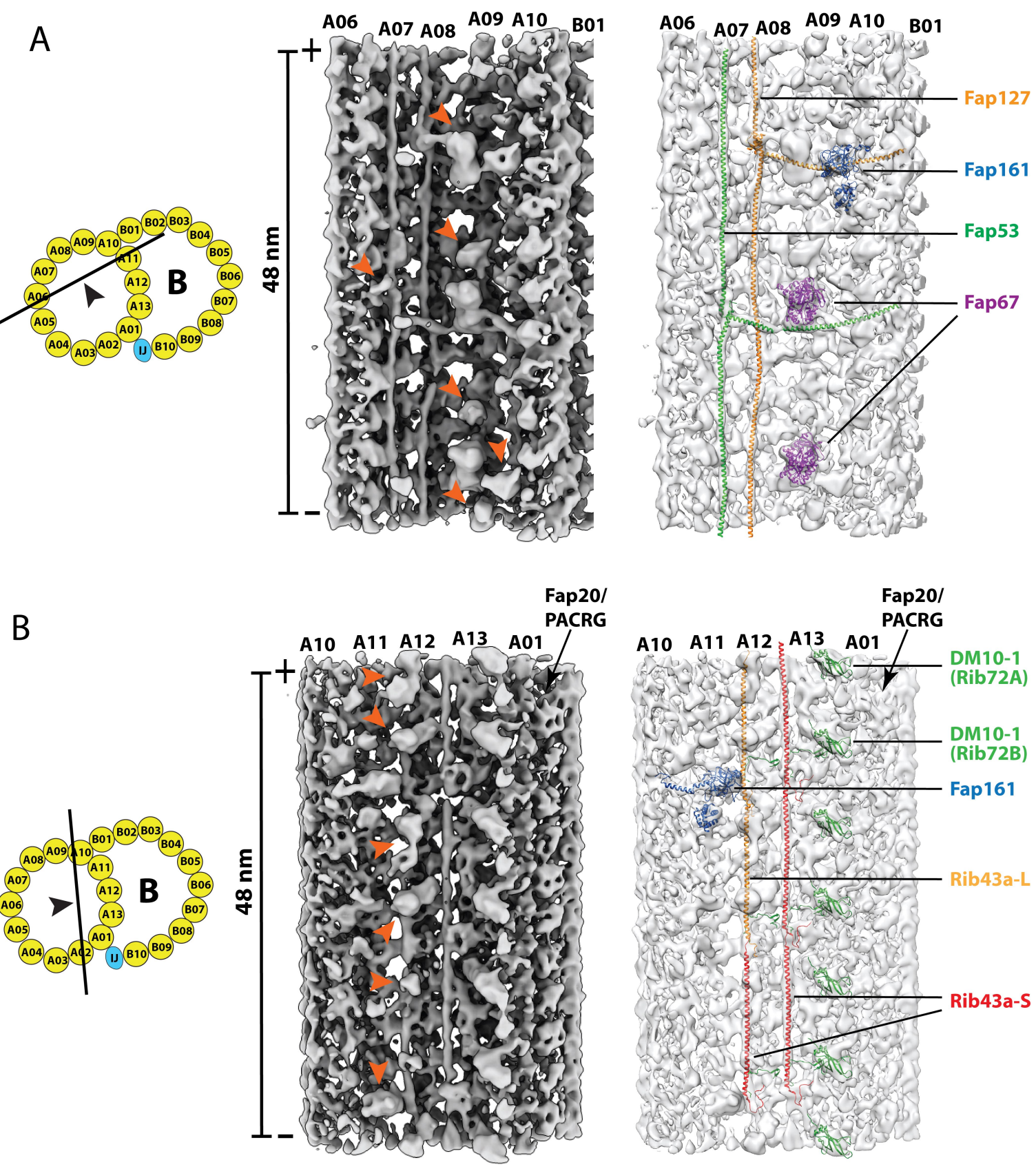


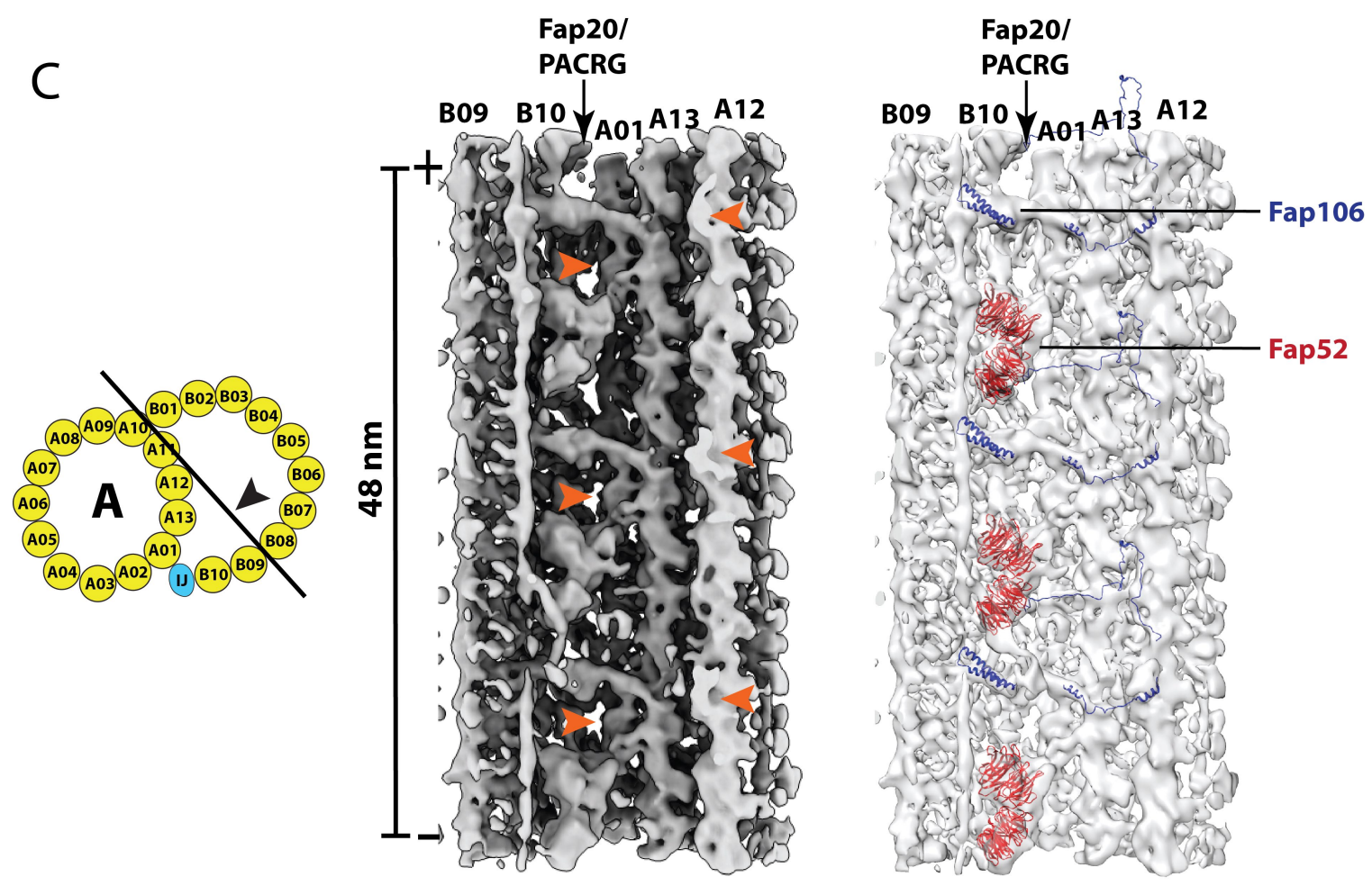

Figure 5. Structure Comparison of the Doublet Microtubule Between Tetrahymena

\section{and Chlamydomonas}

Three regions in the DMT are compared. These are:
A. The A-tubule "seam" region spanning the pf A06 A10.
B. The partition region.
C. The inner junction region.

In each figure: left: a cartoon depicts the viewing direction of the structures in the DMT. middle: the red arrowheads indicate the unidentified MIP densities that are unique to the Tetrahymena DMT. right: the MIPs found in both organisms are indicated. The atomic models from Chlamydomonas are fit into the Tetrahymena density maps in light gray. 


\section{Figure 6}

A
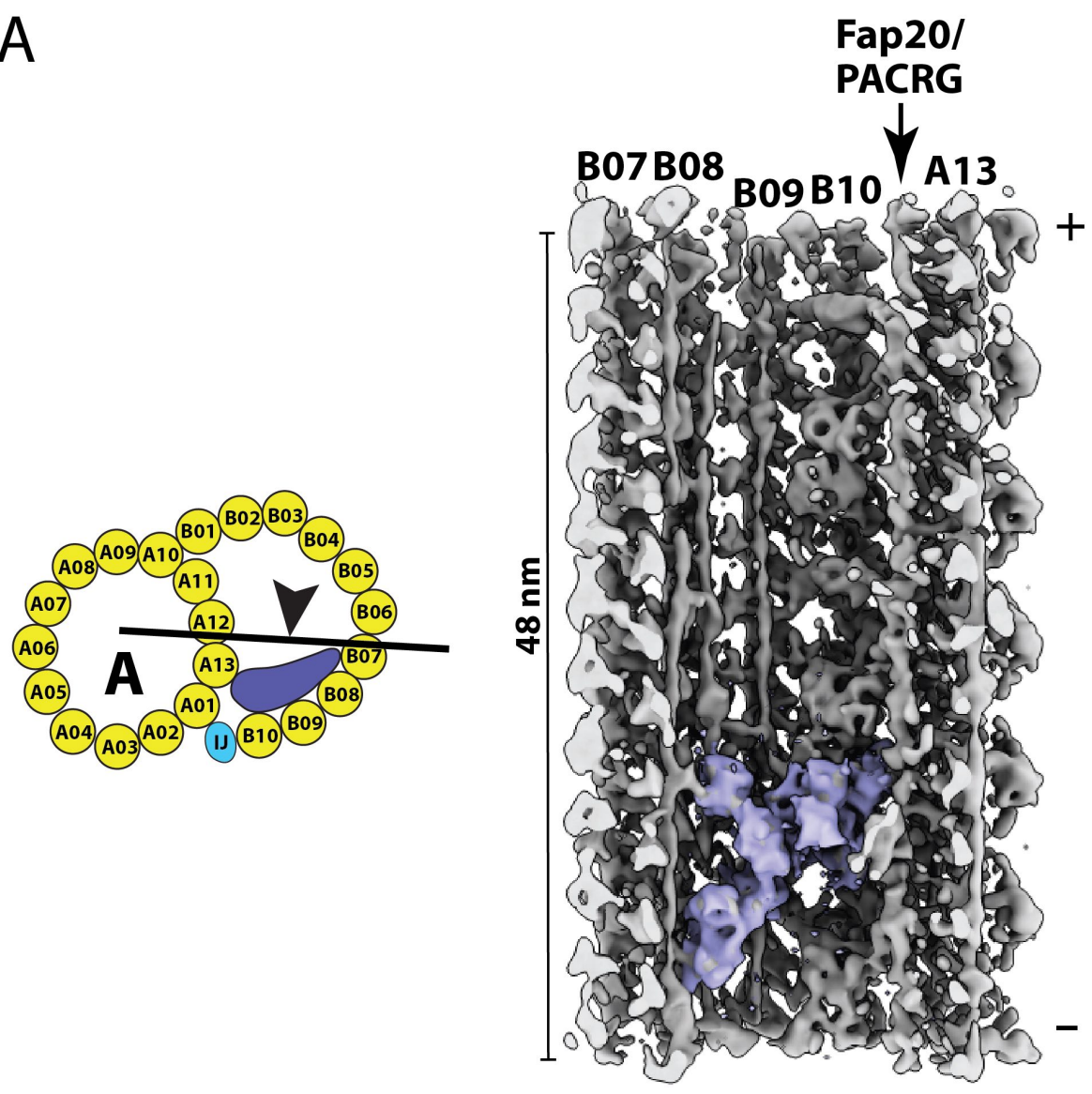

B

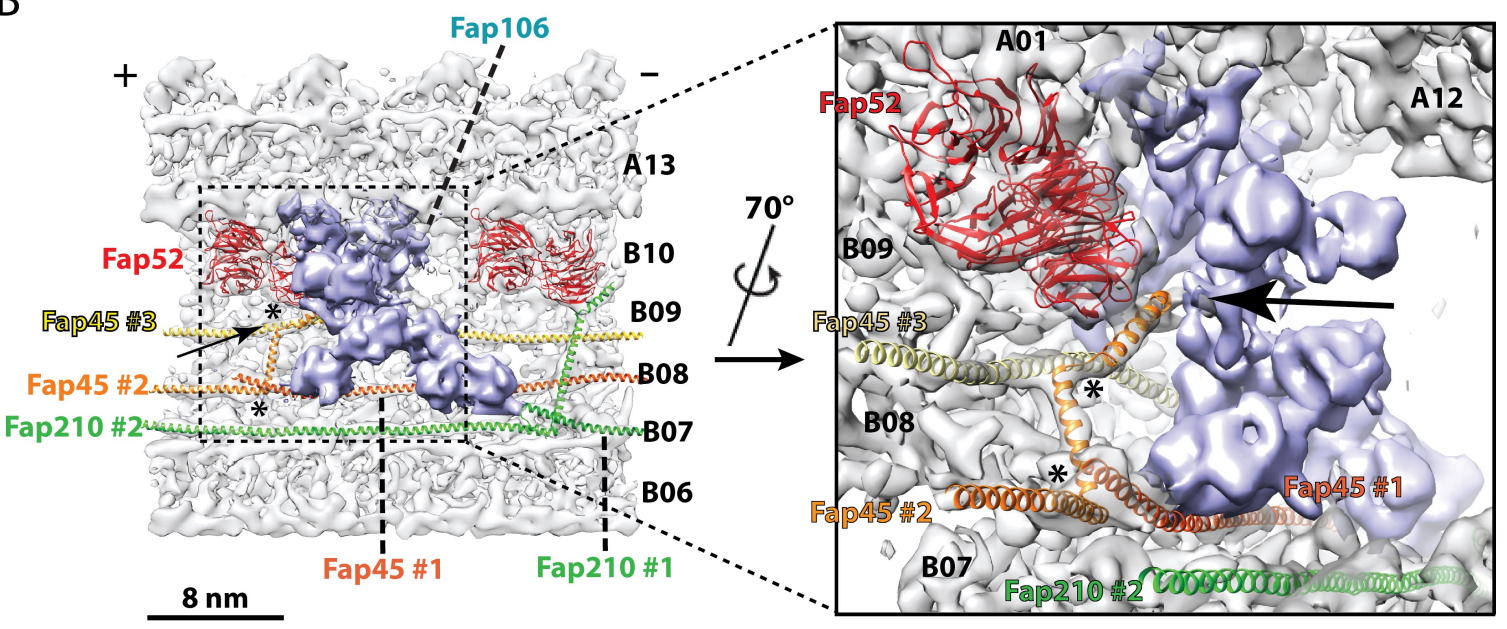

Figure 6. An Unidentified MIP at the Inner Junction of the Doublet Microtubule 
A. An unidentified MIP (royal blue) in the inner junction region of DMT. In the cartoon, an arrowhead indicates the direction of view of the MIP. This multi-domain MIP spans several protofilaments, from B07 to A13, longitudinally repeating every $48 \mathrm{~nm}$.

B. An unidentified MIP (royal blue) makes extensive interactions with multiple protofilaments and other MIPs at the inner junction. On the left panel, the plus end of DMT is towards the left as indicated. Two Fap52 (red) are shown. A Fap106 is behind the unidentified MIP (not shown). Three Fap45 are shown. Fap45 \#1 (dark orange) and Fap45 \#3 (yellow) bind on the cleft between protofilament B07/B08 and B08/B09 respectively. The $\alpha$-helical Fap45 \#2 (orange) initiates from the pf B09, threads through a gap between Fap52 and the unidentified MIP (indicated by an arrow), makes two $~ 90$ degree turns (indicated by two asterisks). Fap45 \#2 interacts with Fap45 \#3 and Fap45 \#1 before landing on the cleft between pf B07/B08. Two Fap210 are shown in green. The inset shows a magnified view of the described interactions among the unidentified MIP (royal blue), Fap45 \#2 and other nearby MIPs. 


\section{Supplemental Figures and Figure Legends}

\section{Figure S1}
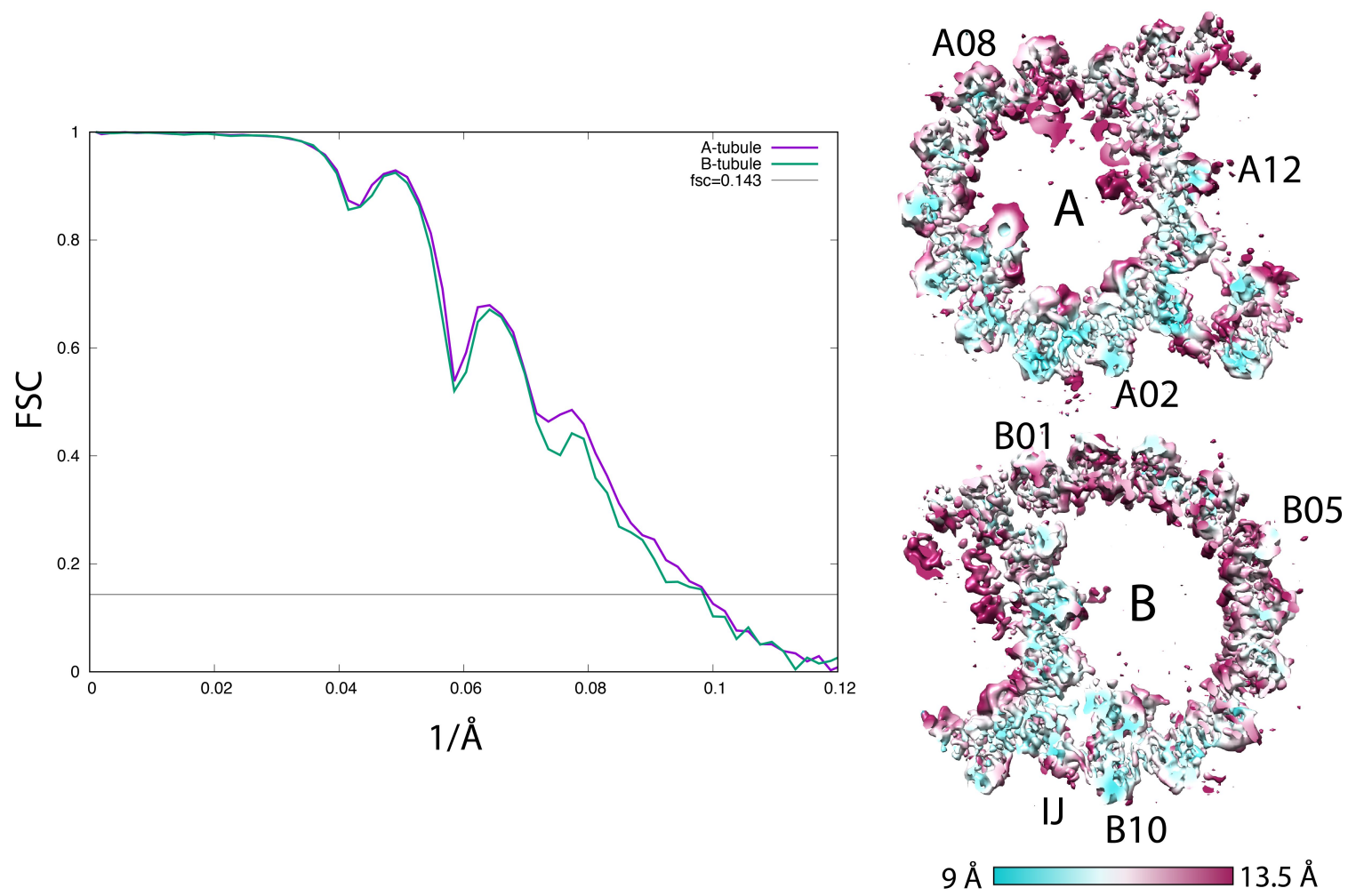

Figure S1 (related to Figure 1). Resolution Assessment by Fourier Shell Correlation

(FSC). Left: the FSC curves of the 16-nm repeat of the wild-type DMT A-tubule (purple, $10.2 \AA$ ) and the B-tubule (green, 10.2 $\AA$ ). Right: the anisotropic local resolution represented by a central section from the structure. 


\section{Figure S2}
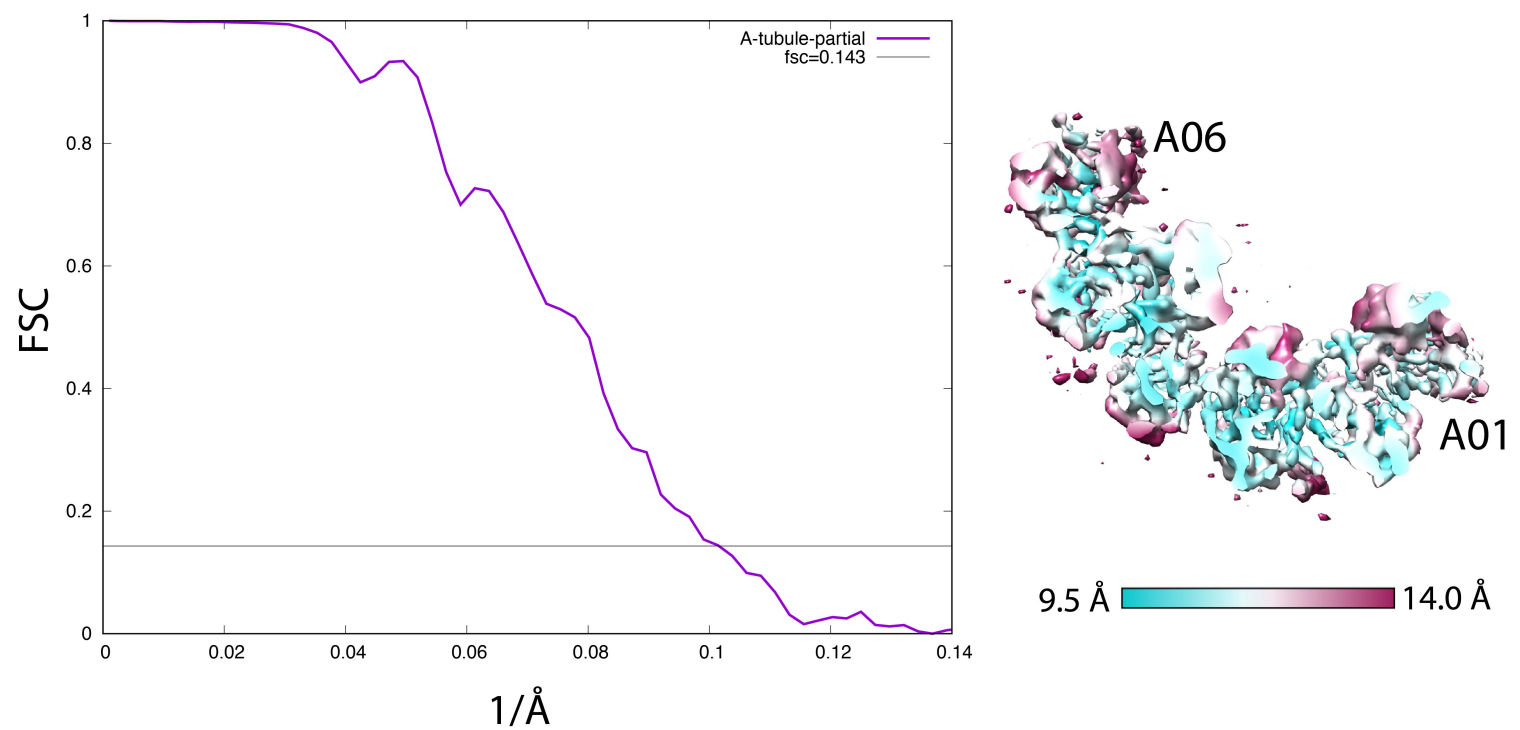

Figure S2 (related to Figure 2). Resolution Assessment by Fourier Shell Correlation (FSC). Left: the FSC curve of the 16-nm repeat of the partial A-tubule from the wildtype, focusing on Rib72A/B (9.9 $\AA)$. Right: the anisotropic local resolution represented by a central section from the structure. 


\section{Figure S4}
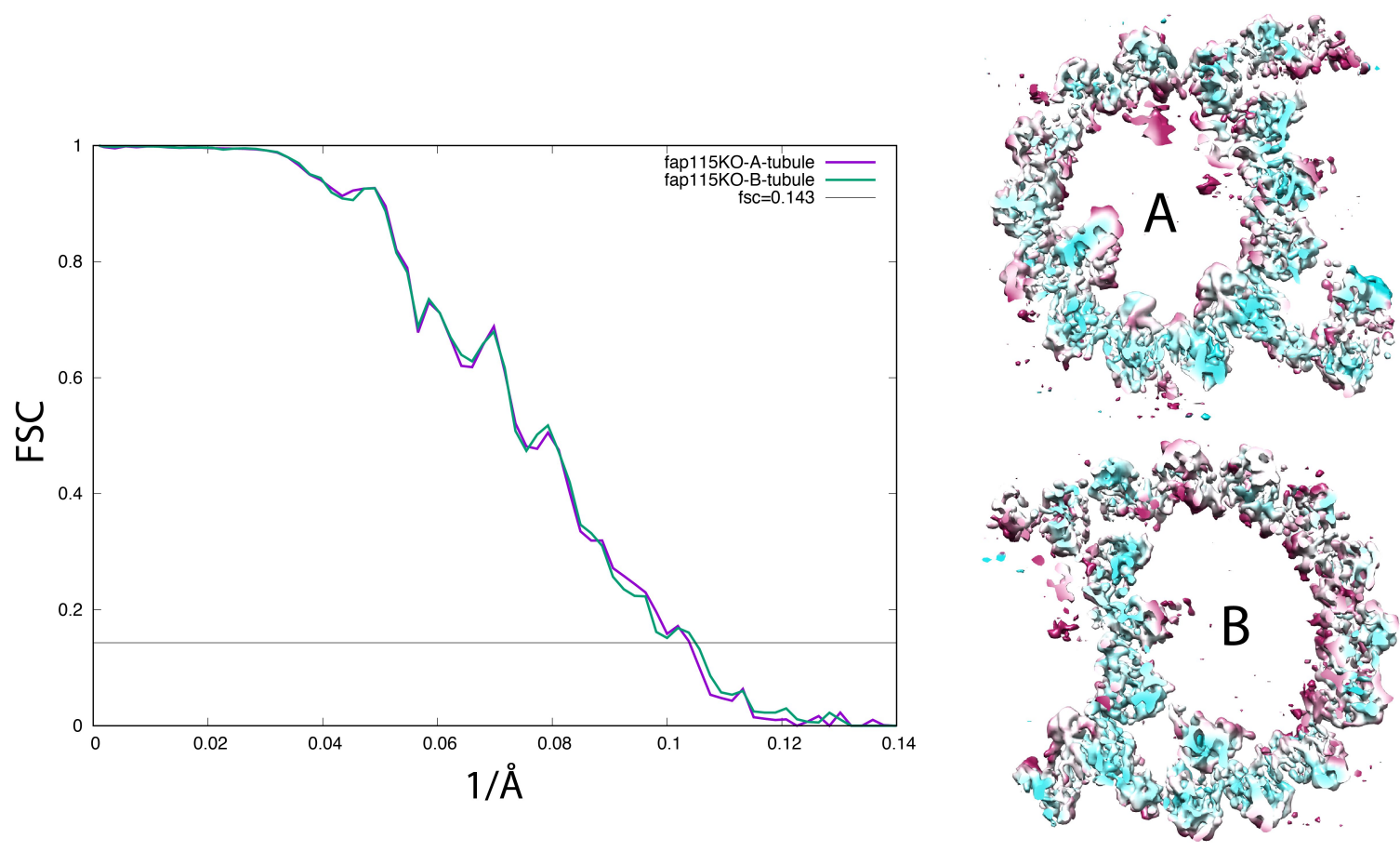

$9 \AA$

$13.5 \AA$

Figure S4A (related to Figure 4A). Resolution Assessment by Fourier Shell

Correlation (FSC). Left: the FSC curves of the 16-nm repeat of the FAP115KO DMT A-

tubule (purple, $9.6 \AA$ ) and the B-tubule (green, 9.6 $)$ ). Right: the anisotropic local

resolution represented by a central section from the structure. 

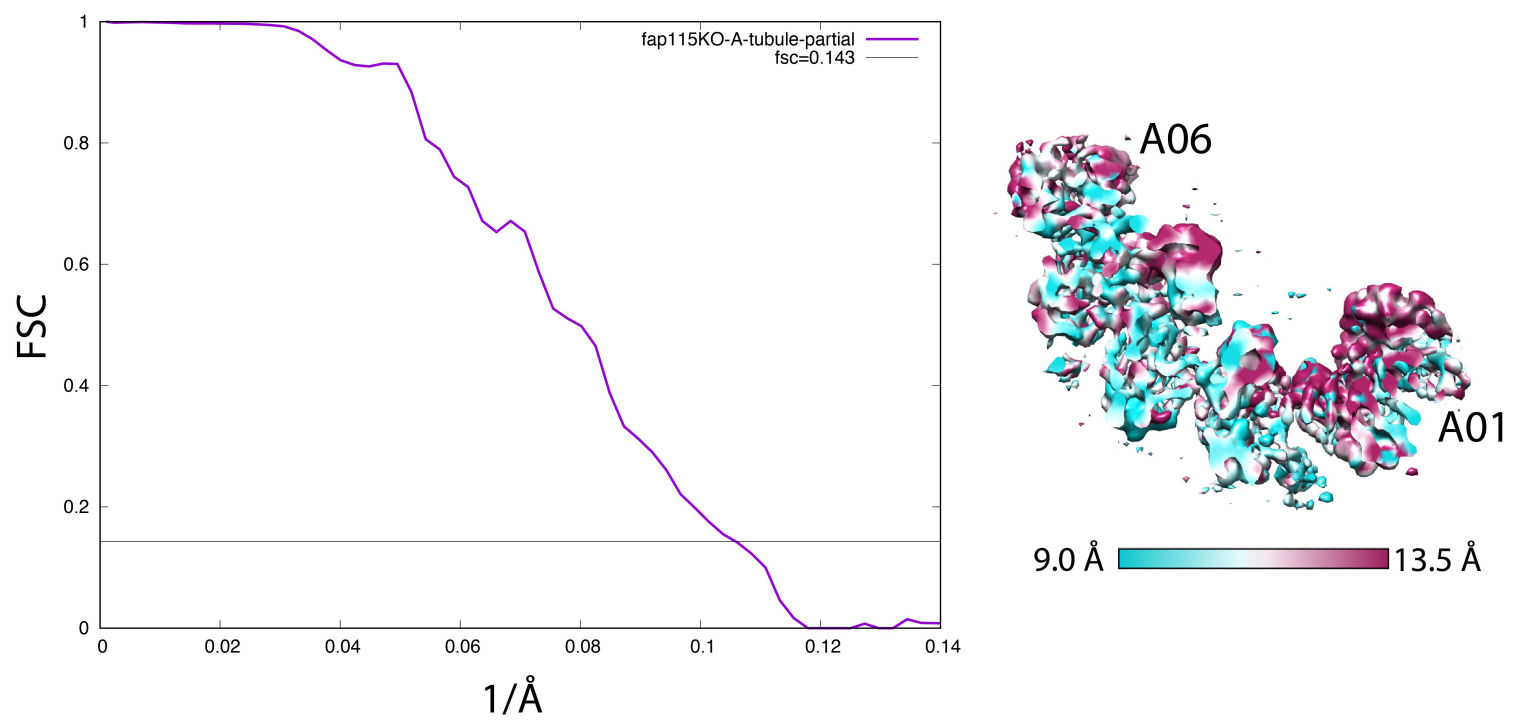

$9.0 \AA$ $13.5 \AA$

Figure S4B (related to Figure 4B). Resolution Assessment by Fourier Shell Correlation (FSC). Left: the FSC curve of the 16-nm repeat of the partial A-tubule from the FAP115KO mutant, focusing on Rib72A/B (9.6 ̊). Right: the anisotropic local resolution represented by a central section from the structure.

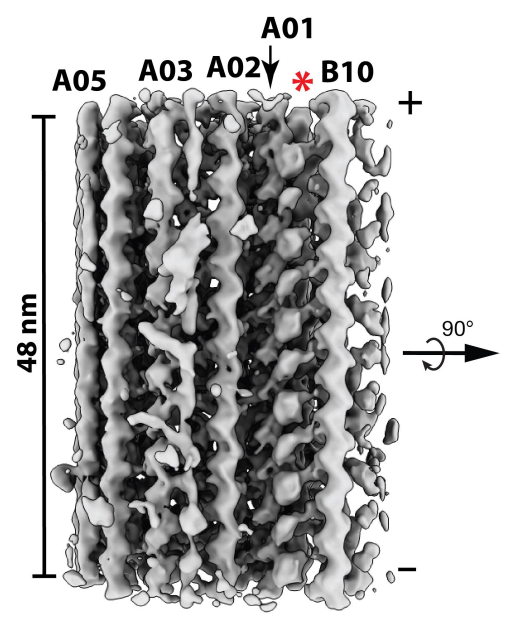

Wild-Type

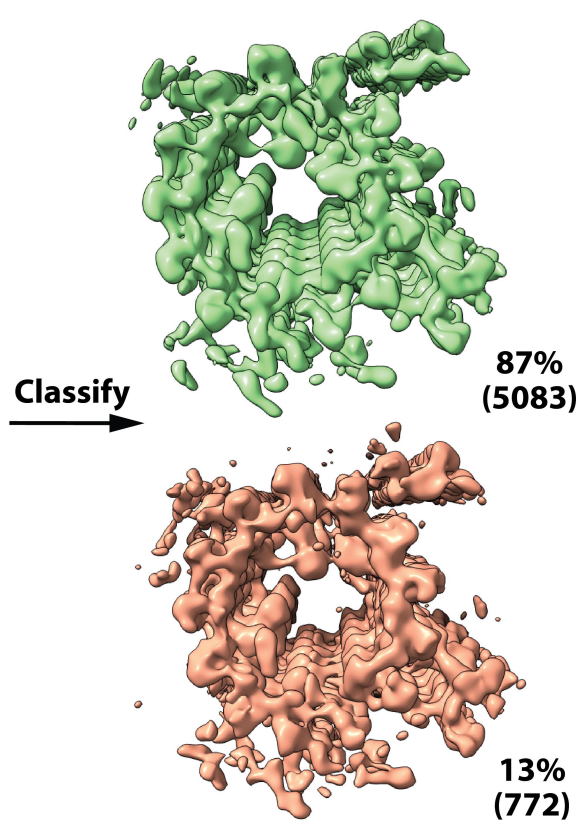


Figure S4C (related to Figure 4C). Classification of the 48-nm Repeat of the A-tubule from the Wild-type. The structure (in gray) is shown in two orthogonal views. A red arrow indicates the location of Fap115. A red asterisk indicates the inner junction protofilament composed of PACRG and Fap20. Classification focusing on the partition pf A11 A13 resulted in two classes as shown on the right - the $87 \%$ intact structure is in green and the $13 \%$ defective structure is in orange. The numbers of subtomograms in each class are indicated.

Figure S4D, E, F

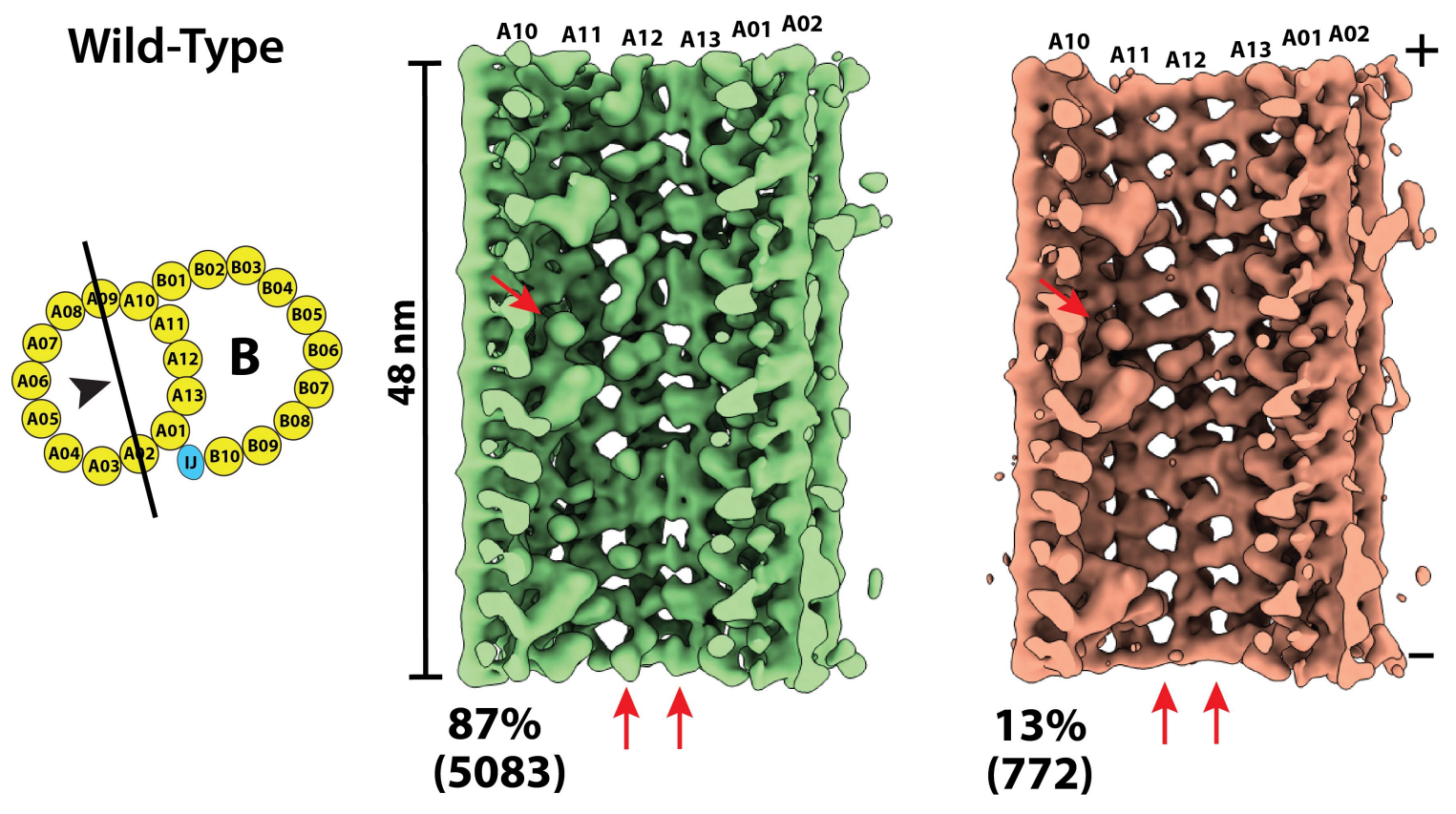



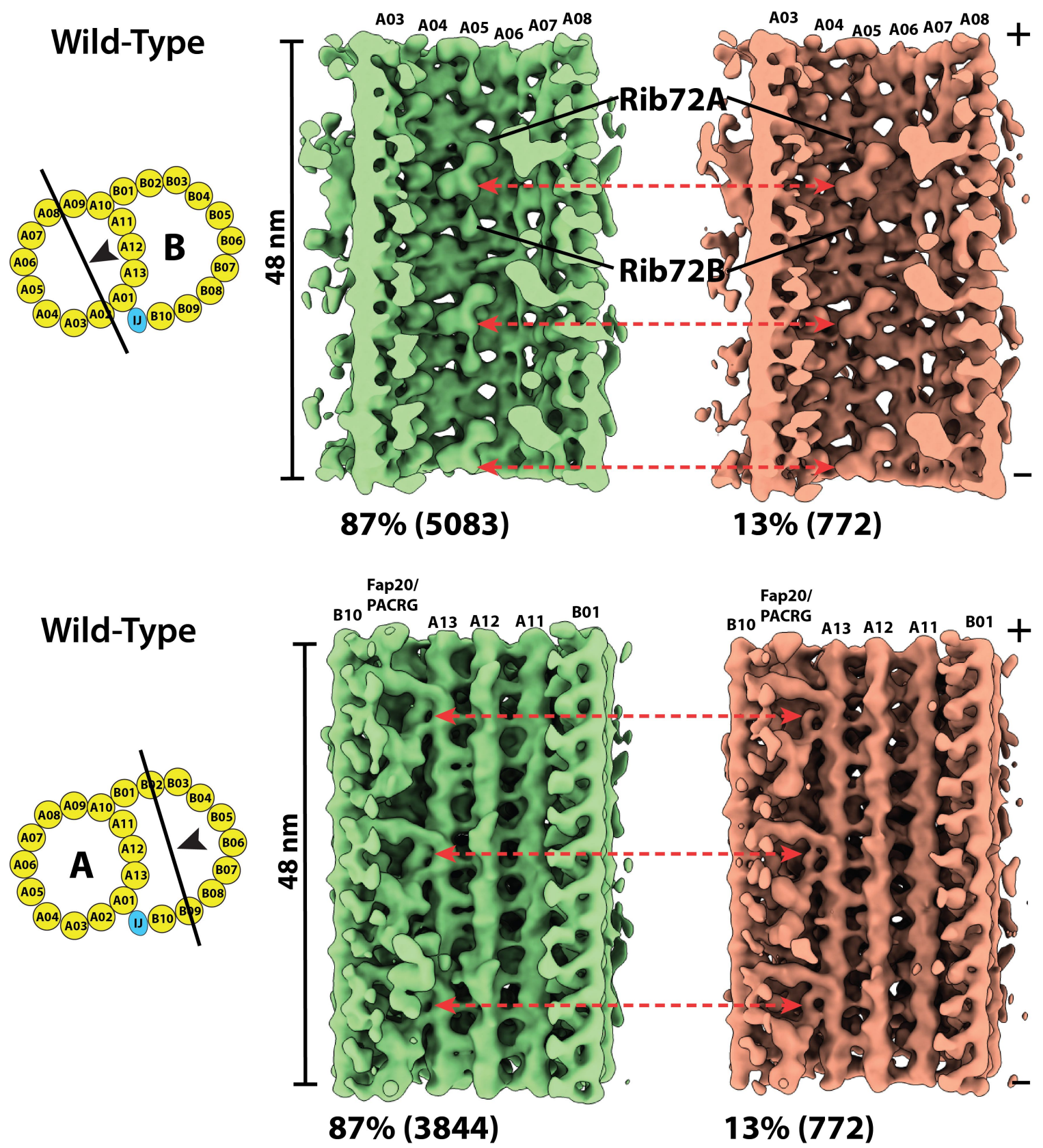

Figure S4D, E, F (related to Figure 4D, E, F). Comparison between two class-averages

from the wild-type DMT in three regions, the partition, the A04A05 and the inner junction region, shows the defects is limited to the partition region. The viewing directions are depicted by the black arrowheads in the cartoons. The two class-averages are displayed side-by-side. The corresponding locations where the structure differences are observed in the FAP115KO mutant are indicated by red arrows. 

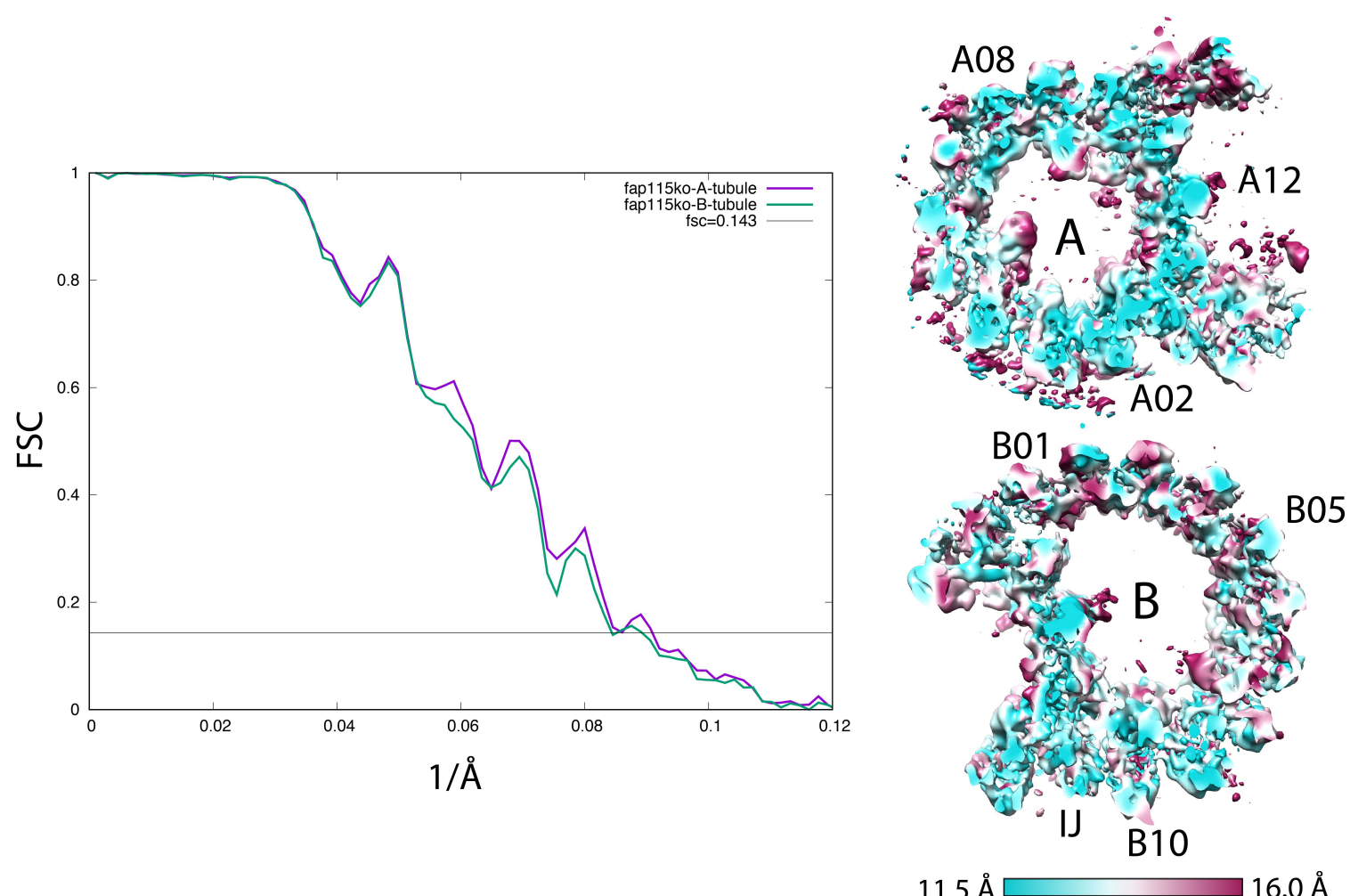

Figure S4G (related to Figure 4C). Resolution Assessments by Fourier Shell

Correlation (FSC). Left: the FSC curves of the 48-nm repeat of the FAP115KO DMT Atubule (purple, 11.8 $\AA$ ) and the B-tubule (green, 12.0 $\AA$ ). Right: the anisotropic local resolution represented by a central section from the structure. 


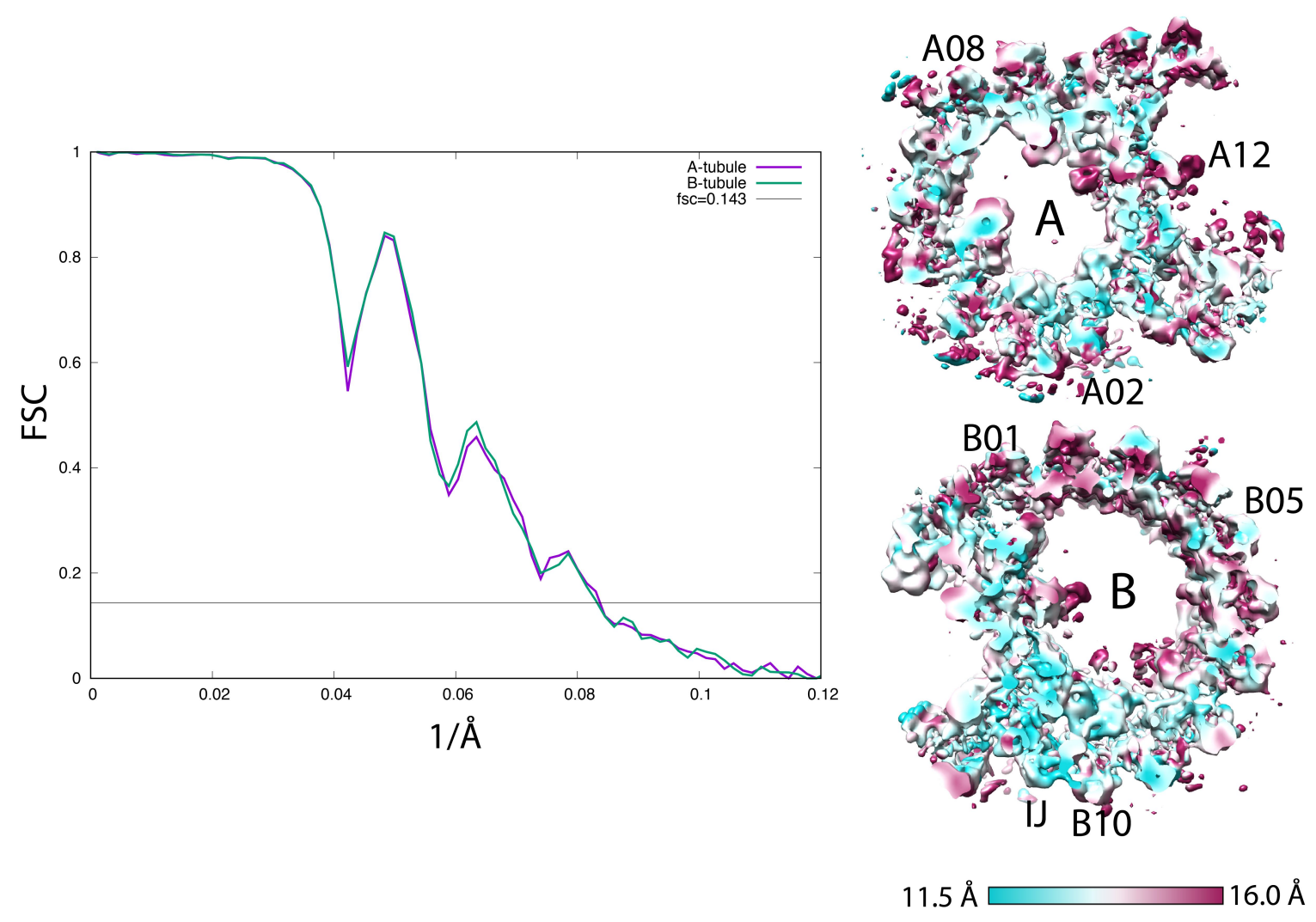

Figure S4H (related to Figure 4C). Resolution Assessments by Fourier Shell

Correlation (FSC). Left: the FSC curves of the 48-nm repeat of the Wild-type DMT A-

tubule (purple, $12.0 \AA$ ) and the B-tubule (green, $12.0 \AA$ ). Right: the anisotropic local

resolution represented by a central section from the structure. 


\section{Figure S5}

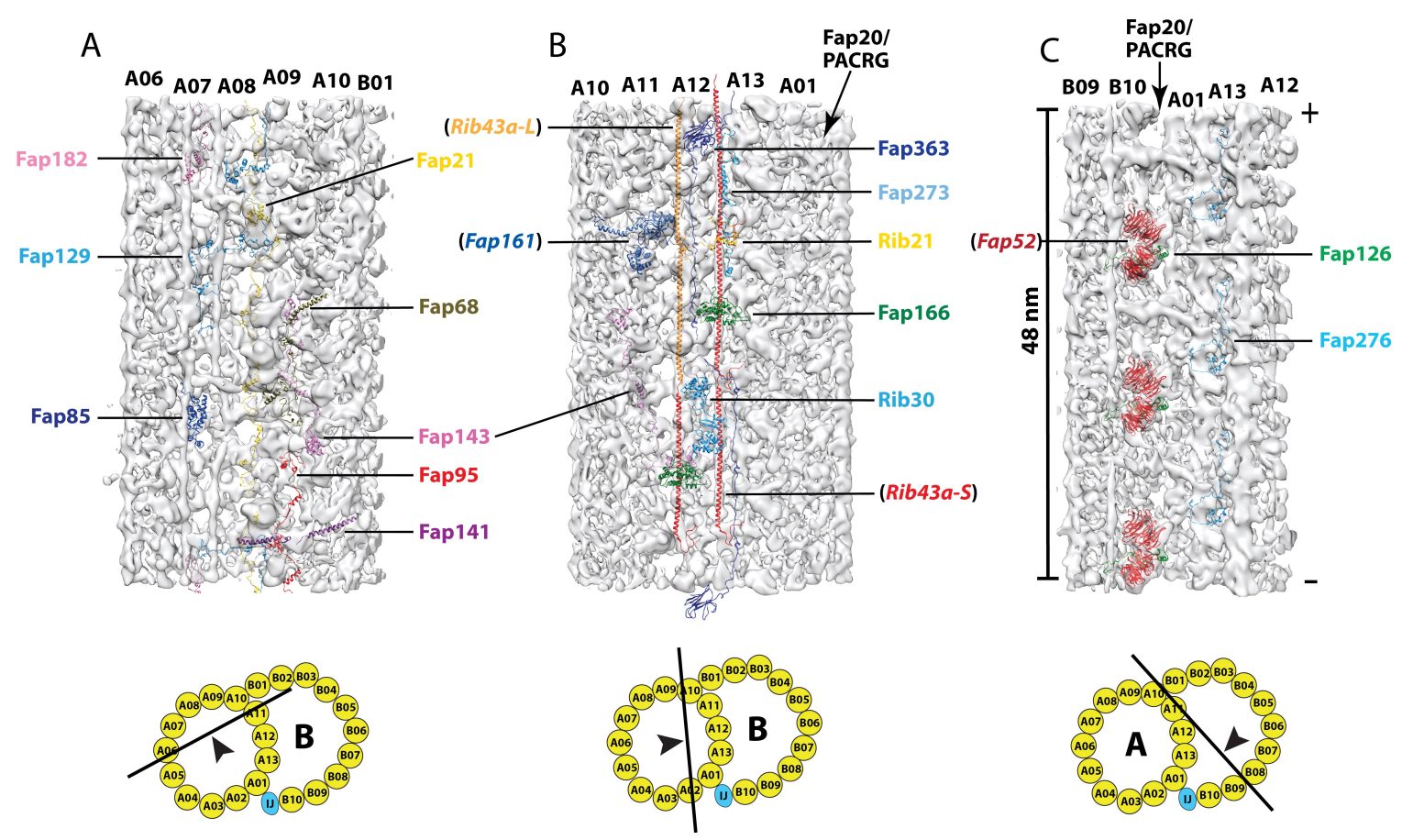

Figure S5 (related to Figure 5A, B, C). A set of MIPs that have been identified in

Chlamydomonas but are absent or could not be identified in Tetrahymena. (A) The Atubule "seam" region spanning pf A06 A10. (B).The partition region. (C) The inner junction region.

The MIPs in parentheses are consensus MIPs found in both organisms, serving as references here.

The arrowheads in the cartoons of DMT indicate the viewing directions. 


\section{Figure S6}
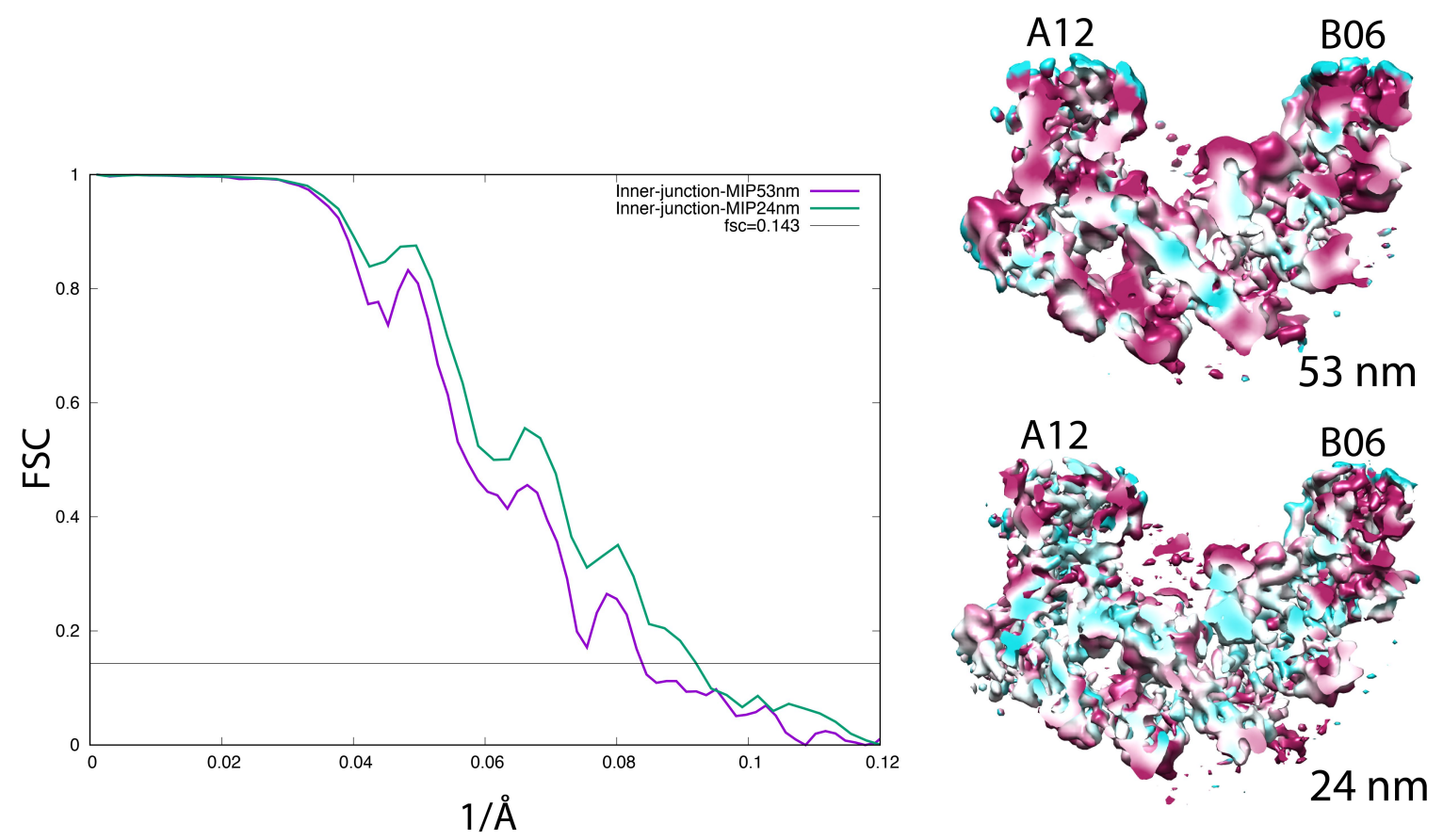

$11.0 \AA$

$15.5 \AA$

Figure S6 (related to Figure 6A, B) Resolution Assessments by Fourier Shell

Correlation (FSC). Left: the FSC curves of the DMT Fragment Focusing on an

Unidentified MIP at the inner junction. DMT length: $48 \mathrm{~nm}$ (purple, $12.0 \AA$ ) and $24 \mathrm{~nm}$

(green, $10.9 \AA$ ). Right: the anisotropic local resolution represented by a central section from the structures. 


\section{Video Legends}

Video 1 (related to Figure 2). An unidentified MIP longitudinally connecting the Cterminus of Rib72A and Rib72B. A surface rendered subtomogram average focusing on the pf A01 A06 segment of A-tubule is shown in grey. The MIP is highlighted in yellow. The DMT longitudinal segment length is $18 \mathrm{~nm}$.

Video 2 (related to Figure 2). An unidentified MIP longitudinally connecting the Cterminus of Rib72A and Rib72B. A surface rendered subtomogram average focusing on the pf A01 A06 segment of A-tubule is shown in transparent light grey The MIP is highlighted in yellow. The models of Rib72A (top) and Rib72B (bottom) are in red.

Video 3 (related to Figure 3). Interaction between Fap115 CTD and $\beta$-tubulins from pfs A02 (left) and A03 (right). The view is from the luminal side of the A-tubule. Fap1 15 is in dark blue. The $\beta$-tubulins are in light blue. The $\alpha$-tubulins are in green in the background.

Video 4 (related to Figure 3). Interaction between Fap115 CTD and $\alpha$-tubulins from pfs A02 (left) and A03 (right). The view is from the luminal side of the A-tubule.

Fap115 is in dark blue. The $\alpha$-tubulins are in green. The $\beta$-tubulins are in light blue in the background. 
Video 5 (related to Figure 6). An unidentified MIP at the inner junction of the DMT. The unidentified MIP is highlighted in royal blue. The DMT longitudinal segment length is $48 \mathrm{~nm}$. The plus end of DMT is to the top.

Video 6 (related to Figure 6). Subtomogram average focusing on the unidentified

MIP at the inner junction. The MIP, highlighted in royal blue, makes extensive interaction with the protofilaments and other MIPs. Two copies of Fap52 are in red. Two copies of Fap210 are in green. Three copies of Fap45 are in dark orange, orange and yellow respectively. The DMT longitudinal segment length is $24 \mathrm{~nm}$. The plus end of DMT is to the left. 


\section{Tables}

Table 1. Summary of Structure from Wild-type and FAP115KO Mutant

\begin{tabular}{|c|c|c|c|c|}
\hline Structure & $\begin{array}{c}\text { Number of } \\
\text { Subtomogram } \\
\end{array}$ & $\begin{array}{l}\text { Resolution } \\
(\AA)\end{array}$ & Description & EMDB ID \# \\
\hline 1 & 16125 & 10.2 & A-tubule, $16 \mathrm{~nm}$, wild-type & EMD-24364 \\
\hline 2 & 15033 & 10.2 & B-tubule, $16 \mathrm{~nm}$, wild-type & EMD-24366 \\
\hline 3 & 13034 & 9.9 & $\begin{array}{l}\text { Partial A-tubule, } 16 \mathrm{~nm} \text {, focusing } \\
\text { on Rib72A/B, wild-type }\end{array}$ & EMD-24367 \\
\hline 4 & 5083 & 12.0 & A-tubule, $48 \mathrm{~nm}$, wild-type & EMD-24368 \\
\hline 5 & 5855 & 12.0 & A-tubule, $48 \mathrm{~nm}$, wild-type & EMD-24370 \\
\hline 6 & 16967 & 9.6 & A-tubule, $16 \mathrm{~nm}, F A P 115 \mathrm{KO}$ & EMD-24371 \\
\hline 7 & 17133 & 9.6 & B-tubule, $16 \mathrm{~nm}, F A P 115 \mathrm{KO}$ & EMD-24372 \\
\hline 8 & 12697 & 9.6 & $\begin{array}{l}\text { Partial A-tubule, } 16 \mathrm{~nm} \text {, } \\
\text { focusing on } \mathrm{Rib} 72 \mathrm{~A} / \mathrm{B} \text {, } \\
\text { FAP1 } 15 \mathrm{KO}\end{array}$ & EMD-24373 \\
\hline 9 & 9502 & 11.8 & A-tubule, $48 \mathrm{~nm}, F A P 115 \mathrm{KO}$ & EMD-24374 \\
\hline 10 & 9502 & 12.0 & B-tubule, $48 \mathrm{~nm}, F A P 115 \mathrm{KO}$ & EMD-24375 \\
\hline 11 & 4960 & 12.0 & $\begin{array}{l}\text { An unknown MIP in inner } \\
\text { junction region, } 48 \mathrm{~nm}\end{array}$ & EMD-24376 \\
\hline 12 & 4960 & 10.9 & $\begin{array}{l}\text { Focusing on an unknown MIP } \\
\text { in inner junction region, } 24 \mathrm{~nm}\end{array}$ & EMD-24377 \\
\hline 13 & - & - & $\begin{array}{l}\text { Composite map combining \#1 } \\
\# 2,16 \mathrm{~nm} \text {, wild-type }\end{array}$ & EMD-24379 \\
\hline 14 & - & - & $\begin{array}{l}\text { Composite map combining } \# 4 \\
\# 5,48 \mathrm{~nm} \text {, wild-type }\end{array}$ & EMD-24380 \\
\hline 15 & - & - & $\begin{array}{l}\text { Composite map combining \#6 } \\
\quad \# 7,16 \mathrm{~nm}, F A P 115 \mathrm{KO}\end{array}$ & EMD-24381 \\
\hline 16 & - & - & $\begin{array}{l}\text { Composite map combining \#9 } \\
\quad \# 10,48 \mathrm{~nm}, F A P 115 \mathrm{KO}\end{array}$ & EMD-24382 \\
\hline
\end{tabular}


Table 2. Comparison of MIP Structure between Tetrahymena t. and Chlamydomonas $r$.

\begin{tabular}{|c|c|}
\hline $\begin{array}{l}19 \text { MIPs found in both Tetrahymena } t \text {. and } \\
\text { Chlamydomonas } r \text {. }\end{array}$ & $\begin{array}{l}\text { Rib72, Fap115, Fap67, Fap53, Fap127, } \\
\text { Fap161, Rib43, Fap20 (fly), PACRG, } \\
\text { Fap52, Fap106, Fap45, Fap210, Fap112, } \\
\text { CCDC39/Fap59, CCDC40/Fap172, DC1, } \\
\text { DC2, DC3 }\end{array}$ \\
\hline $\begin{array}{l}19 \text { MIPs identified in Chlamydomonas } r \text {. } \\
\text { but possibly absent in Tetrahymena } t \text {. }\end{array}$ & $\begin{array}{l}\text { Fap222, Fap252, Fap21, Fap68, Fap107, } \\
\text { Fap85, Fap90, Fap95, Fap129, Fap141, } \\
\text { Fap143, Fap182 (mice), Fap166, Fap273, } \\
\text { Fap363, Rib21, Rib30, Fap126 (mice), } \\
\text { Fap276 }\end{array}$ \\
\hline $\begin{array}{l}19 \text { Unidentified MIPs or complexes found } \\
\text { in Tetrahymena } t \text {. whose density are } \\
\text { missing in Chlamydomonas } r \text {. and their } \\
\text { atomic models are lacking, }\end{array}$ & $\begin{array}{l}1 \text { in pf A04 A05 region } \\
6 \text { in pf A06 A10 region } \\
6 \text { in partition region } \\
3 \text { in inner junction region } \\
3 \text { fMIPs in pf B03 B06 }\end{array}$ \\
\hline
\end{tabular}

Note:

The MIPs highlighted in red cause ciliopathy in human or phenotypic defects in other metazoa as indicated. These are based on a summary in (Ma et al, 2019) and references cited within. 


\section{Materials and Methods}

\section{Isolation of Axoneme from the Tetrahymena Strains}

The wild-type (B2086.2) Tetrahymena strain was obtained from the Tetrahymena Stock Center (Cornell University, Ithica, NY). All strains were grown in the standard medium 2\% SPP $\left(2 \%\right.$ protease peptone, $0.1 \%$ yeast extract, $0.2 \%$ glucose, $\left.0.003 \% \mathrm{FeCl}_{3}\right)$ at $30^{\circ} \mathrm{C}$.

The FAP115 knockout strain was generated as described previously (Fabritius et al., 2020).

To isolate axoneme from the cells, cells grown in $500 \mathrm{ml}$ 2\% SPP media were harvested by spinning down at $1100 x g$ for 5 minutes in room temperature. Cells were washed with Tris- $\mathrm{HCl}(\mathrm{pH} 7.5)$ and span down again at 1100xg for 5 minutes. Cells were suspended in pH-Shock Buffer (10 mM Tris, $\mathrm{pH} 7.5,50 \mathrm{mM}$ sucrose, $10 \mathrm{mM} \mathrm{CaCl}_{2}, 0.33 \%$ Proteinase Inhibitor Cocktail (Sigma)). 0.5 M acetic acid was added to lower the $\mathrm{pH}$ to 4.3. After one minute, the $\mathrm{pH}$ was brought back to 7.5 by adding $1 \mathrm{M} \mathrm{KOH}$ (Sigma). The deflagellated cells were spun at $1500 \mathrm{xg}$ for 5 minutes, in $4{ }^{\circ} \mathrm{C}$. The supernatant was centrifuged twice at $1860 \mathrm{xg}$ for 5 minutes in $4{ }^{\circ} \mathrm{C}$. The cilia in the supernatant were pelleted by spinning at $10,000 \mathrm{xg}$ for 15 minutes. The cilia pellet was resuspended in cold HMEEK buffer (30 mM HEPES, 25 mM KCl, 5 mM MgSO $4,0.1$ mM EDTA, 1 mM K-EGTA). 1\% Igepal CA 630 (Sigma) was added to demembrane the axoneme. After rotating for 20 minutes in $4{ }^{\circ} \mathrm{C}$, the sample was centrifuged at $10,000 \times \mathrm{xg}$ for 10 minutes. The pellet containing the axoneme was carefully resuspended in cold HMEEK buffer.

\section{Electron Cryo-Tomography Data Collection}


To make grids for cryoET, the isolated ciliary axonemes were mixed with $10 \mathrm{~nm}$ colloid gold (Sigma). 4 l sample was applied onto Quantifoil grid Cu/Rh 200 R2/2 (Quantifoil, Inc), and was flash frozen into liquid ethane using a Vitrobot (FEI, Inc). Tomography tilt series were collected on a field emission gun (FEG) microscope (Titan Krios, FEI, Inc) running at 300kV. A Bio-Quantum GIF post-column energy filter (Gatan, Inc) was used during data collection. The slit width was set at $20 \mathrm{eV}$. SerialEM (Mastronarde, 2005) was used for collecting tomography tilt series at a nominal magnification of 33,000. Images were recorded on a K3 direct electron detector (Gatan, Inc) in super-resolution and dose-fractionation mode. The effective physical pixel size on image was $2.65 \AA$. The dose rate was set at 20 electron/pixel/second. The specimen was tilted in a bi-directional scheme, starting from zero degree, first tilted towards $-60^{\circ}$, followed by a second half from $+2^{\circ}$ to $+60^{\circ}$, in $2^{\circ}$ increment per step. The total accumulative dose on the sample was limited to 80 electron $/ \AA^{2}$.

\section{Data Processing for Subtomogram Averaging and Model Building}

For tomogram reconstruction and subtomogram averaging, the dose-fractionated movie at each tilt in the tilt series was corrected of motion and summed using MotionCor2 (Zheng et al., 2017). The tilt series were aligned based on the gold beads as fiducials by using IMOD (Kremer et al., 1996) and TomoAlign (Fernandez et al., 2018). The contrast transfer function for each tilt series was determined and corrected by TomoCTF (Fernández et al., 2006). The tomograms were reconstructed by TomoRec (Fernandez et al., 2019) taking into account of the beam-induced sample motion during data collection. Total 51 wild-type axoneme tomograms from 49 tilt series and 65 fap $115 \mathrm{KO}$ axoneme 
tomograms from 64 tilt series were selected for reconstruction and subtomogram averaging.

To identify the DMT for subtomogram averaging, the 6xbinned tomograms were used. The center of DMT and the approximate orientation of the axoneme relative to the tilt axis were manually annotated in a Spider metadata file (Frank et al., 1996). The initial subtomogram alignment and average were carried out in 2xbinned format (pixel size 5.30 $\AA$ ). The longitudinal segment length of axoneme DMT in subtomogram was limited to 24 $\mathrm{nm}$ and $50 \%$ overlapping with neighboring segments. Without using any external reference, the subtomogram alignment was carried out by a program MLTOMO implemented in the Xmipp software package (Scheres et al., 2009).

Since DMT from axoneme was a continuous filament up to several microns in length, after obtaining initial alignment parameters, a homemade program RANSAC was used to detect and remove any alignment outliers and to impose the continuity constraint on the neighboring segments. It corrected the misaligned subtomograms by regression. MLTOMO and Relion (Bharat and Scheres, 2016) were extensively used for focused classification of the subtomograms. This was critical for determining the correct periodicity of the MIPs and for identifying structure defects or heterogeneity in the DMT. In the focused classification, a particular MIP structure in a subregion of DMT was chosen for discerning different classes. Specifically, in the pf A01 A06 region, the unidentified MIP with $16 \mathrm{~nm}$ periodicity that crosslinked Rib72A/B was used as a fiducial to identify the out-of-register classes; in the A-tubule seam region, two copies of 
Fap67 were used as fiducials for identifying the local $48 \mathrm{~nm}$ periodicity; in the inner junction region, the unidentified MIP spanning multiple pfs with $48 \mathrm{~nm}$ periodicity was used as a fiducial. The focused classification results were further confirmed by positions of other MIPs in the region. Since the assembly of DMT is a coherent process, the relative position of a particular MIP to other MIPs will be consistent. Once a MIP's periodicity was determined, the out-of-register subtomograms were re-centered and reextracted. This was followed by combining all subtomograms for the next round of refinement.

For final refinement and averaging, the subtomograms were re-extracted from tomogram volumes in unbinned format (pixel size 2.65 Å). During the extraction, a customized, soft-edged and cylindrical shaped binary mask, centered on a particular region or the structure of interest in the DMT, was imposed onto the extracted subtomograms. This was carried out by the program Spider (Frank et al., 1996). Except the refinement of the unidentified MIP in the inner junction where the DMT longitudinal length was arbitrarily set at $24 \mathrm{~nm}$, in all other cases, the extracted DMT segment length was set to be 18 or 53 $\mathrm{nm}$, containing a full repeat of the structure that was in either 16 or $48 \mathrm{~nm}$ periodicity as determined by the focused classification. The re-extracted and masked subtomograms, containing only the structure of interest, for example the pf A01 A06 region in the Atubule or the inner junction region, were aligned in a two-independent-datasets scheme implemented in Relion (Scheres and Chen, 2012). The refinement took into account doseweighting and thickness weighting (Bharat and Scheres, 2016). The overall resolutions were reported based on the Fourier Shell Correlation (FSC) cutoff at 0.143 (Rosenthal 
and Henderson, 2003). The local resolution assessments were carried out by the program blocres in Bsoft (Heymann and Belnap, 2007). This is particularly useful for assessing the resolution anisotropy of large structures such as the DMT.

A composite map is useful for putting together individual component maps, often in higher resolution, in the context of large assemblage. The composite maps were generated in UCSF Chimera (Pettersen et al., 2004). First, the component maps were aligned based on their mutual overlapping regions by using UCSF Chimera's "fit-in-map" function. This was followed by taking maximum density values from the overlapping voxels and combining the component maps into a composite map. UCSF Chimera and ChimeraX (Pettersen et al., 2021) were used for visualization, model building and for recording images and videos.

The pseudo-atomic models for different parts of the DMT were built in Chimera or PyMol by fitting previously published atomic models into the subtomogram averaging density maps.

16 structures, including their EMDB access codes are summarized in Table 1. 


\section{References}

Akella JS, Wloga D, Kim J, Starostina NG, Lyons-Abbott S, Morrissette NS, Dougan ST, Kipreos ET, Gaertig J. 2010. MEC-17 is an $\alpha$-tubulin acetyltransferase. Nature 467:218-222. doi:10.1038/nature09324

Avidor-Reiss T, Maer AM, Koundakjian E, Polyanovsky A, Keil T, Subramaniam S, Zuker CS. 2004. Decoding Cilia Function Defining Specialized Genes Required for Compartmentalized Cilia Biogenesis. Cell 117:527-539. doi:10.1016/s00928674(04)00412-x

Bharat TAM, Scheres SHW. 2016. Resolving macromolecular structures from electron cryo-tomography data using subtomogram averaging in RELION. Nat Protoc 11:2054-2065. doi:10.1038/nprot.2016.124

Bornens M. 2018. Cell polarity: having and making sense of direction —on the evolutionary significance of the primary cilium/centrosome organ in Metazoa. Open Biol 8:180052. doi:10.1098/rsob.180052

Carbajal-González BI, Heuser T, Fu X, Lin J, Smith BW, Mitchell DR, Nicastro D. 2013. Conserved structural motifs in the central pair complex of eukaryotic flagella. Cytoskeleton 70:101-120. doi:10.1002/cm.21094

Carvalho-Santos Z, Azimzadeh J, Pereira-Leal JoséB, Bettencourt-Dias M. 2011. Tracing the origins of centrioles, cilia, and flagella. J Cell Biology 194:165-175. doi:10.1083/jcb.201011152

Cavalier-Smith T. 2002. The phagotrophic origin of eukaryotes and phylogenetic classification of Protozoa. Int J Syst Evol Micr 52:297-354. doi:10.1099/0020771352-2-297

Diener DR, Yang P, Geimer S, Cole DG, Sale WS, Rosenbaum JL. 2011. Sequential assembly of flagellar radial spokes. Cytoskeleton 68:389-400. doi:10.1002/cm.20520

Dymek EE, Lin J, Fu G, Porter ME, Nicastro D, Smith EF. 2019. PACRG and FAP20 form the inner junction of axonemal doublet microtubules and regulate ciliary motility. Mol Biol Cell 30:1805-1816. doi:10.1091/mbc.e19-01-0063

Eshun-Wilson L, Zhang R, Portran D, Nachury MV, Toso DB, Löhr T, Vendruscolo M, Bonomi M, Fraser JS, Nogales E. 2019. Effects of $\alpha$-tubulin acetylation on microtubule structure and stability. Proc National Acad Sci 116:201900441. doi:10.1073/pnas.1900441116 
Fabritius AS, Bayless BA, Li S, Stoddard D, Heydeck W, Ebmeier CC, Anderson L, Gunnels T, Nachiappan C, Whittall JB, Old W, Agard DA, Nicastro D, Winey M. 2020. Proteomic analysis of microtubule inner proteins (MIPs) in Rib72 null Tetrahymena cells reveals functional MIPs. Biorxiv 2020.10.02.324467. doi:10.1101/2020.10.02.324467

Fernandez J-J, Li S, Agard DA. 2019. Consideration of sample motion in cryotomography based on alignment residual interpolation. J Struct Biol 205:1-6. doi:10.1016/j.jsb.2019.01.005

Fernandez J-J, Li S, Bharat TAM, Agard DA. 2018. Cryo-tomography tilt-series alignment with consideration of the beam-induced sample motion. J Struct Biol 202:200-209. doi:10.1016/j.jsb.2018.02.001

Fernández JJ, Li S, Crowther RA. 2006. CTF determination and correction in electron cryotomography. Ultramicroscopy 106:587-596. doi:10.1016/j.ultramic.2006.02.004

Fourniol FJ, Sindelar CV, Amigues B, Clare DK, Thomas G, Perderiset M, Francis F, Houdusse A, Moores CA. 2010. Template-free 13-protofilament microtubule-MAP assembly visualized at $8 \AA$ resolution. J Cell Biology 191:463-470.

doi:10.1083/jcb.201007081

Frank J, Radermacher M, Penczek P, Zhu J, Li Y, Ladjadj M, Leith A. 1996. SPIDER and WEB: Processing and Visualization of Images in 3D Electron Microscopy and Related Fields. J Struct Biol 116:190-199. doi:10.1006/jsbi.1996.0030

Gaertig J, Cruz MA, Bowen J, Gu L, Pennock DG, Gorovsky MA. 1995. Acetylation of lysine 40 in alpha-tubulin is not essential in Tetrahymena thermophila. J Cell Biology 129:1301-1310. doi:10.1083/jcb.129.5.1301

Gonsales MC, Ribeiro PAO, Betting LE, Alvim MKM, Guerreiro CM, Yasuda CL, Gitaí DLG, Cendes F, Lopes-Cendes I. 2020. Revisiting the clinical impact of variants in EFHC1 in patients with different phenotypes of genetic generalized epilepsy. Epilepsy Behav 112:107469. doi:10.1016/j.yebeh.2020.107469

Heymann JB, Belnap DM. 2007. Bsoft: Image processing and molecular modeling for electron microscopy. J Struct Biol 157:3-18. doi:10.1016/j.jsb.2006.06.006

Howes SC, Geyer EA, LaFrance B, Zhang R, Kellogg EH, Westermann S, Rice LM, Nogales E. 2017. Structural differences between yeast and mammalian microtubules revealed by cryo-EM. J Cell Biol 216:2669-2677. doi:10.1083/jcb.201612195

Ichikawa M, Khalifa AAZ, Kubo S, Dai D, Basu K, Maghrebi MAF, Vargas J, Bui KH. 2019. Tubulin lattice in cilia is in a stressed form regulated by microtubule inner proteins. Proc National Acad Sci 116:19930-19938. doi:10.1073/pnas.1911119116 
Ichikawa M, Liu D, Kastritis PL, Basu K, Hsu TC, Yang S, Bui KH. 2017.

Subnanometre-resolution structure of the doublet microtubule reveals new classes of microtubule-associated proteins. Nat Commun 8:15035. doi:10.1038/ncomms15035

Khalifa AAZ, Ichikawa M, Dai D, Kubo S, Black C, Peri K, McAlear TS, Veyron S, Yang SK, Vargas J, Bechstedt S, Trempe J-F, Bui KH. 2020. The inner junction complex of the cilia is an interaction hub that involves tubulin post-translational modifications. Elife 9:e52760. doi:10.7554/elife.52760

King SM. 2006. Axonemal protofilament ribbons, DM10 domains, and the link to juvenile myoclonic epilepsy. Cell Motil Cytoskel 63:245-253. doi:10.1002/cm.20129

Kirima J, Oiwa K. 2017. Flagellar-associated protein FAP85 is a microtubule inner protein that stabilizes microtubules. Cell Struct Funct 43:17023. doi:10.1247/csf.17023

Kremer JR, Mastronarde DN, McIntosh JR. 1996. Computer Visualization of ThreeDimensional Image Data Using IMOD. J Struct Biol 116:71-76. doi:10.1006/jsbi.1996.0013

Kumar S, Stecher G, Suleski M, Hedges SB. 2017. TimeTree: A Resource for Timelines, Timetrees, and Divergence Times. Mol Biol Evol 34:1812-1819. doi:10.1093/molbev/msx116

Li JB, Gerdes JM, Haycraft CJ, Fan Y, Teslovich TM, May-Simera H, Li H, Blacque OE, Li L, Leitch CC, Lewis RA, Green JS, Parfrey PS, Leroux MR, Davidson WS, Beales PL, Guay-Woodford LM, Yoder BK, Stormo GD, Katsanis N, Dutcher SK. 2004. Comparative Genomics Identifies a Flagellar and Basal Body Proteome that Includes the BBS5 Human Disease Gene. Cell 117:541-552. doi:10.1016/s00928674(04)00450-7

Lin J, Nicastro D. 2018. Asymmetric distribution and spatial switching of dynein activity generates ciliary motility. Science 360:eaar1968. doi:10.1126/science.aar1968

Lin J, Yin W, Smith MC, Song K, Leigh MW, Zariwala MA, Knowles MR, Ostrowski LE, Nicastro D. 2014. Cryo-electron tomography reveals ciliary defects underlying human RSPH1 primary ciliary dyskinesia. Nat Commun 5:5727. doi:10.1038/ncomms6727

Ma M, Stoyanova M, Rademacher G, Dutcher SK, Brown A, Zhang R. 2019. Structure of the Decorated Ciliary Doublet Microtubule. Cell 179:909-922.e12. doi:10.1016/j.cell.2019.09.030

Mastronarde DN. 2005. Automated electron microscope tomography using robust prediction of specimen movements. J Struct Biol 152:36-51. doi:10.1016/j.jsb.2005.07.007 
Maurer SP, Fourniol FJ, Bohner G, Moores CA, Surrey T. 2012. EBs Recognize a Nucleotide-Dependent Structural Cap at Growing Microtubule Ends. Cell 149:371382. doi:10.1016/j.cell.2012.02.049

Mitchell DR. 2016. Evolution of Cilia. Csh Perspect Biol 9:a028290. doi:10.1101/cshperspect.a028290

Mitchison TJ, Mitchison HM. 2010. How cilia beat. Nature 463:308-309. doi:10.1038/463308a

Nicastro D, Fu X, Heuser T, Tso A, Porter ME, Linck RW. 2011. Cryo-electron tomography reveals conserved features of doublet microtubules in flagella. Proc National Acad Sci 108:E845-E853. doi:10.1073/pnas.1106178108

Nicastro D, Schwartz C, Pierson J, Gaudette R, Porter ME, McIntosh JR. 2006. The Molecular Architecture of Axonemes Revealed by Cryoelectron Tomography. Science 313:944-948. doi:10.1126/science.1128618

Norrander JM, deCathelineau AM, Brown JA, Porter ME, Linck RW. 2000. The Rib43a Protein Is Associated with Forming the Specialized Protofilament Ribbons of Flagellar Microtubules inChlamydomonas. Mol Biol Cell 11:201-215. doi:10.1091/mbc.11.1.201

Oda T, Yanagisawa H, Kamiya R, Kikkawa M. 2014. A molecular ruler determines the repeat length in eukaryotic cilia and flagella. Science 346:857-860.

doi:10.1126/science. 1260214

Ostrowski LE, Blackburn K, Radde KM, Moyer MB, Schlatzer DM, Moseley A, Boucher RC. 2002. A Proteomic Analysis of Human Cilia Identification of Novel Components*S. Mol Cell Proteomics 1:451-465. doi:10.1074/mcp.m200037-mcp200

Owa M, Uchihashi T, Yanagisawa H, Yamano T, Iguchi H, Fukuzawa H, Wakabayashi K, Ando T, Kikkawa M. 2019. Inner lumen proteins stabilize doublet microtubules in cilia and flagella. Nat Commun 10:1143. doi:10.1038/s41467-019-09051-X

Pazour GJ, Agrin N, Leszyk J, Witman GB. 2005. Proteomic analysis of a eukaryotic cilium. J Cell Biology 170:103-113. doi:10.1083/jcb.200504008

Pettersen EF, Goddard TD, Huang CC, Couch GS, Greenblatt DM, Meng EC, Ferrin TE. 2004. UCSF Chimera - A visualization system for exploratory research and analysis. J Comput Chem 25:1605-1612. doi:10.1002/jcc.20084

Pettersen EF, Goddard TD, Huang CC, Meng EC, Couch GS, Croll TI, Morris JH, Ferrin TE. 2021. UCSF ChimeraX: Structure visualization for researchers, educators, and developers. Protein Sci 30:70-82. doi:10.1002/pro.3943 
Pigino G, Maheshwari A, Bui KH, Shingyoji C, Kamimura S, Ishikawa T. 2012. Comparative structural analysis of eukaryotic flagella and cilia from Chlamydomonas, Tetrahymena, and sea urchins. J Struct Biol 178:199-206.

doi:10.1016/j.jsb.2012.02.012

Qin H, Diener DR, Geimer S, Cole DG, Rosenbaum JL. 2004. Intraflagellar transport (IFT) cargo. J Cell Biology 164:255-266. doi:10.1083/jcb.200308132

Rai A, Liu T, Katrukha EA, Estévez-Gallego J, Paterson I, Díaz JF, Kapitein LC, Moores CA, Akhmanova A. 2021. Microtubule lattice defects promote catastrophes. Biorxiv 2021.02.11.430743. doi:10.1101/2021.02.11.430743

Reiter JF, Leroux MR. 2017. Genes and molecular pathways underpinning ciliopathies. Nat Rev Mol Cell Bio 18:533-547. doi:10.1038/nrm.2017.60

Rosenbaum JL, Witman GB. 2002. Intraflagellar transport. Nat Rev Mol Cell Bio 3:813825. doi:10.1038/nrm952

Rosenthal PB, Henderson R. 2003. Optimal Determination of Particle Orientation, Absolute Hand, and Contrast Loss in Single-particle Electron Cryomicroscopy. J Mol Biol 333:721-745. doi:10.1016/j.jmb.2003.07.013

Sale WS, Satir P. 1976. Splayed Tetrahymena cilia. A system for analyzing sliding and axonemal spoke arrangements. J Cell Biology 71:589-605. doi:10.1083/jcb.71.2.589

Satir P, Christensen ST. 2007. Overview of Structure and Function of Mammalian Cilia. Annu Rev Physiol 69:377-400. doi:10.1146/annurev.physiol.69.040705.141236

Satir P, Mitchell DR, Jékely G. 2008. Chapter 3 How Did the Cilium Evolve? Curr Top Dev Biol 85:63-82. doi:10.1016/s0070-2153(08)00803-x

Schaedel L, John K, Gaillard J, Nachury MV, Blanchoin L, Théry M. 2015. Microtubules self-repair in response to mechanical stress. Nat Mater 14:1156-1163. doi: $10.1038 /$ nmat4396

Scheres SHW, Chen S. 2012. Prevention of overfitting in cryo-EM structure determination. Nat Methods 9:853-854. doi:10.1038/nmeth.2115

Scheres SHW, Melero R, Valle M, Carazo J-M. 2009. Averaging of Electron Subtomograms and Random Conical Tilt Reconstructions through Likelihood Optimization. Structure 17:1563-1572. doi:10.1016/j.str.2009.10.009

Smith JC, Northey JGB, Garg J, Pearlman RE, Siu KWM. 2005. Robust Method for Proteome Analysis by MS/MS Using an Entire Translated Genome: Demonstration on the Ciliome of Tetrahymena t hermophila. J Proteome Res 4:909-919. doi:10.1021/pr050013h 
Song K, Shang Z, Fu X, Lou X, Grigorieff N, Nicastro D. 2020. In situ structure determination at nanometer resolution using TYGRESS. Nat Methods 17:201-208. doi:10.1038/s41592-019-0651-0

Stepanek L, Pigino G. 2016. Microtubule doublets are double-track railways for intraflagellar transport trains. Science 352:721-724. doi:10.1126/science.aaf4594

Stoddard D, Zhao Y, Bayless BA, Gui L, Louka P, Dave D, Suryawanshi S, Tomasi RFX, Dupuis-Williams P, Baroud CN, Gaertig J, Winey M, Nicastro D. 2018. Tetrahymena RIB72A and RIB72B are microtubule inner proteins in the ciliary doublet microtubules. Mol Biol Cell 29:2566-2577. doi:10.1091/mbc.e18-06-0405

Sui H, Downing KH. 2006. Molecular architecture of axonemal microtubule doublets revealed by cryo-electron tomography. Nature 442:475-478. doi:10.1038/nature04816

Suzuki T, Delgado-Escueta AV, Aguan K, Alonso ME, Shi J, Hara Y, Nishida M, Numata T, Medina MT, Takeuchi T, Morita R, Bai D, Ganesh S, Sugimoto Y, Inazawa J, Bailey JN, Ochoa A, Jara-Prado A, Rasmussen A, Ramos-Peek J, Cordova S, Rubio-Donnadieu F, Inoue Y, Osawa M, Kaneko S, Oguni H, Mori Y, Yamakawa K. 2004. Mutations in EFHC1 cause juvenile myoclonic epilepsy. Nat Genet 36:842849. doi:10.1038/ng1393

Suzuki T, Inoue I, Yamakawa K. 2020. Epilepsy protein Efhc1/myoclonin1 is expressed in cells with motile cilia but not in neurons or mitotic apparatuses in brain. Sci Rep-uk 10:22076. doi:10.1038/s41598-020-79202-4

Xu Z, Schaedel L, Portran D, Aguilar A, Gaillard J, Marinkovich MP, Théry M, Nachury MV. 2017. Microtubules acquire resistance from mechanical breakage through intralumenal acetylation. Science 356:328-332. doi:10.1126/science.aai8764

Yanagisawa H, Mathis G, Oda T, Hirono M, Richey EA, Ishikawa H, Marshall WF, Kikkawa M, Qin H. 2014. FAP20 is an inner junction protein of doublet microtubules essential for both the planar asymmetrical waveform and stability of flagella in Chlamydomonas. Mol Biol Cell 25:1472-1483. doi:10.1091/mbc.e13-08-0464

Zhang R, Alushin GM, Brown A, Nogales E. 2015. Mechanistic Origin of Microtubule Dynamic Instability and Its Modulation by EB Proteins. Cell 162:849-859. doi:10.1016/j.cell.2015.07.012

Zheng SQ, Palovcak E, Armache J-P, Verba KA, Cheng Y, Agard DA. 2017. MotionCor2: anisotropic correction of beam-induced motion for improved cryoelectron microscopy. Nat Methods 14:331-332. doi:10.1038/nmeth.4193 